\title{
ChemSpaX: Exploration of chemical space by automated functionalization of molecular scaffold
}

\author{
Adarsh V. Kalikadien ${ }^{1 \odot}$, Evgeny A. Pidko ${ }^{1 *}$, and Vivek Sinha ${ }^{*}{ }^{*}$ \\ ${ }^{1}$ Inorganic Systems Engineering, Department of Chemical Engineering, Faculty of \\ Applied Sciences, Delft University of Technology, Van der Maasweg 9, 2629 HZ, \\ Delft, The Netherlands.
}

June 7, 2021

\begin{abstract}
Exploration of the local chemical space of molecular scaffolds is a highly promising route to discover novel molecules with desired structure and function. Considerable success has been achieved in data-driven exploration of the chemical space of small organic molecules for photovoltaic and pharmaceutical applications. However, structure-property databases spanning the local chemical space of transition metal (TM) complexes, which are widely used in homogeneous catalysis, are not available. In this work a Python-based workflow, ChemSpaX, that is aimed at automating local chemical space exploration for any type of molecule, is introduced with special emphasis on TM catalysts. ChemSpaX uses 3D information to place functional groups on an input structure. The newly placed substituents are selectively optimized using a computationally cheap force-field optimization method to yield 3D molecular geometries. In representative applications of ChemSpaX, it is shown that the generated structures have a reasonable quality for usage in computational screening applications. We demonstrate ChemSpaX via three catalytic applications involving functionalization of 1) various intermediates in (potential) (de)hydrogenation catalysis via $\mathrm{Mn}$ - and Ru-based pincer complexes 2) cobalt porphyrin complexes 3) a bipyridyl functionalized cobalt-porphyrin trapped in a $\mathrm{M}_{2} \mathrm{~L}_{4}$ type cage complex. The quality of geometries was assessed using energetic (error in net reaction energy) and spatial (root mean squared deviation) metrics by comparison against DFT and GFN2-xTB calculations. Results show that geometries generated by ChemSpaX often have performance close to GFN2-xTB optimized geometries although several challenging cases are also identified. Descriptors such as the Gibbs free energy of reaction and HOMO-LUMO gap, that can be used in data-driven design and discovery of catalysts, were selected and studied in more detail. ChemSpaX is open-source and aims to bolster and democratize the efforts of the scientific community towards data-driven chemical discovery.
\end{abstract}

Keywords: catalysis; data-driven chemistry; chemical space; transition metal complexes; open source;

\section{Introduction}

Chemical research has been driven by the ability and the need to create molecular scaffolds with desired (bio)chemical functions. Experimental chemistry, largely guided by intuition, chemical knowledge, and serendipity has been reasonably successful in discovering functional molecular scaffolds which can be improved further. For example, catalysts are decorated with diverse functional groups to explore their activity and stability, and devise possible strategies for improvement. 
Although in vitro functionalization can reveal chemical design principles that underlie high activity, selectivity and stability, it is time and resource intensive. Computational molecular design has emerged as particularly promising in this regard, thanks to recent advances in quantum chemical methods and high performance computing $[1,2,3,4,5,6,7,8]$. High-throughput computational methods can guide towards development of highly effective functionalization strategies by systematically exploring geometries in the local chemical space of a given scaffold [9].

The chemical space is vast and a global exploration is difficult $[10,11]$. Therefore, machine learning $(\mathrm{ML})$ and other cost-effective computational methods are attractive solutions to navigate the chemical space in the search of novel molecules and materials [12]. A data-driven statistical approach, rooted in quantum and statistical mechanics (QM and SM) is needed to explore and understand the chemical space [13]. Such an approach is strongly dependent on the availability of trustworthy structure property databases (SPDBs). For (small) organic molecules, reliable data sets such as the GDB datasets $(11,13$ and 17) exist which are being used for diverse data-driven chemical discovery applications $[14,15,16,17,18,19,20,21]$. In contrast to small organic molecules, development of data-driven approaches have proven more challenging for transition metal complexes (TMCs). TMCs are often used as bio-inspired homogeneous catalysts which account for over $15 \%$ of all industrial catalytic processes and enable key catalytic transformations such as for pharmaceuticals, fine chemicals, and energy applications [22, 23, 24, $25,26,27,28,29]$. Recent studies have revealed the promise of data-driven quantum chemical methods to understand structure-function relations in TMCs [30, 31, 32, 33, 34, 35]. Availability of structure-property data from QM calculations and/or experiments is central to the success of data-driven chemical approaches. SPDBs of homogeneous catalysts are currently not available [28]. SPDBs with a dense representation of the chemical space of catalytic scaffolds can help discover design principles leading to development of sustainable catalytic systems for various applications [36, 37, 28, 38, 39, 40,41]. Representations of the molecular structure is of high importance in SPDBs. Several approaches have been developed in this regard recently. For organic molecules string based SMILES representation is a popular and effective approach to encode the molecular structure of organic molecules [42, 43]. Generally SMILES encoding does not work well for TMCs and generation of 3D geometries using SMILES is an active research area $[44,45]$.

Low dimensional encoding of molecular representations of TMCs, while certainly desirable for ML applications, co-exists with the need to know the 3D geometry due to high structural sensitivity in catalysis. Therefore, automated approaches to rapidly generate accurate 3D molecular representations are also needed. Some automated tools have recently been developed to generate molecular geometries of TMCs [46, 47, 48, 49, 50, 51, 52, 32]. While these tools represent a significant progress in automated and rapid generation of molecular geometries, we sought to develop an easy-to-use tool which can quickly create reasonably accurate molecular geometries in the local chemical space of a given molecular scaffold.

In this manuscript we present ChemSpaX, a Python-based workflow that can be used for automated exploration of the chemical space of molecules. The exploration is done by automated placement of substituents on a given molecular scaffold while maintaining the quality of the initial scaffold. This process is supposed to simulate the workflow used by experimental chemists in which a molecular structure is tested and explored by placing various functional groups. Several molecular scaffolds are already present in literature and can be used for an automated exploration of local chemical space. If a particular complex is known for its high catalytic activity, the 3D coordinates of this complex can be used as a starting point for exploration in the neighbourhood of its chemical space. The user has full control of the placement of substituent groups and can guide the exploration of the local chemical space. A general overview of the approach used in 
ChemSpaX is given in Figure 1.

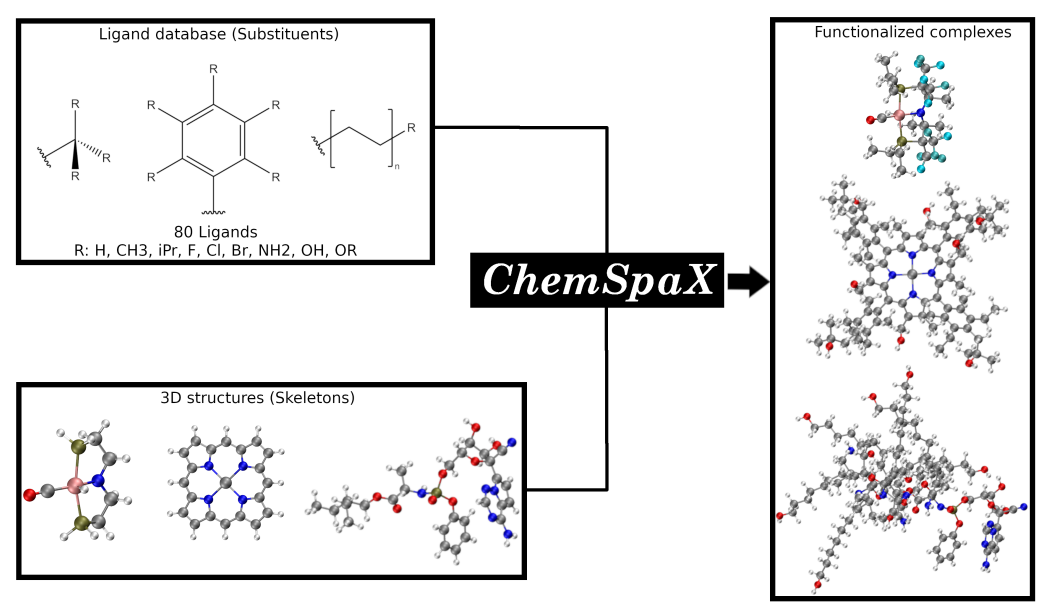

Figure 1: A general overview of the approach used in ChemSpaX. The local chemical space of a pre-optimized input molecular scaffold can be explored by automatically placing ligands from a pre-defined ligand library. Color code used for elements: gray $=\mathrm{C}$, white $=\mathrm{H}$, red $=\mathrm{O}$, pink $=\mathrm{Ru}$, dark-blue $=\mathrm{N}$ and turquoise $=\mathrm{F}$.

The computational methods employed in this manuscript are presented in the next section. A description of the code implementation to develop ChemSpaX follows. Subsequently, representative applications of ChemSpaX are presented. The first representative application involves Ru and $\mathrm{Mn}$ catalysts based on pincer ligands. Pincer ligands have emerged as versatile ligand platform enabling a plethora of catalytic reactions [53, 54, 55, 56]. First, the functionalization of a Ru complex derived from the commercially available Ru-MACHO [25] is described. TMCs based on the MACHO ligand framework have shown versatile activity in catalyzed (de)hydrogenation reactions $[57,58,59,60,61,62]$. Analyzing the chemical space of the Ru-MACHO catalytic scaffold can be a valuable asset for multiple applications. Next, the functionalization of Mn-pincer complexes as potential (de)hydrogenation catalysts is studied [63]. With this application the chemical space of an earth-abundant alternative to RuPNP is explored. Manganese is known to be a cheap, abundant and biocompatible alternative to precious-metal catalysts $[64,59,65,66]$. The quality of geometries generated by ChemSpaX is compared against higher-level DFT and GFN2-xTB methods.

Secondly, we apply ChemSpaX to generate a database of $\sim 1100$ functionalized Cobalt Porphyrin (referred to as 'Co porphyrin' in the rest of this manuscript) complexes. Co porphyrins exist as stable metalloradicals and are used to catalyze carbene and nitrene transfer reactions $[67,68,69,70]$. The generated database is used to investigate quality of geometries generated by ChemSpaX and the propagation of errors upon creation of larger geometries. Additionally, a comparison of electronic properties calculated by GFN2-xTB and DFT is made.

Finally, the functionalization of a bipyridyl functionalized Cobalt-porphyrin trapped in a $\mathrm{M}_{2} \mathrm{~L}_{4}$ type cage complex (referred to as ' $M_{2} L_{4}$ cage' in the rest of this manuscript) is presented. This cage complex confines the Co porphyrin catalyst, which can lead to changed catalyst properties 
$[71,72,70]$. The quality of ChemSpaX generated $M_{2} L_{4}$ cage geometries is compared against semi-empirical and force-field based XTB optimization methods. This case shows how ChemSpaX can be used to functionalize diverse and challenging molecular scaffolds.

\section{Computational methods}

\subsection{Open Babel}

Conversions between MDL Molfile and XYZ format were done using Open Babel [73, 74]. Open Babel was also used to perform Generalized Amber Force Field (GAFF), and the Universal Force Field (UFF) optimizations [75, 76].

\subsection{Semiempirical tight-binding}

Grimme lab's $\times$ TB package (version 6.3.3) was used for semi-empirical tight-binding calculations [77]. The GFN2-xTB, and GFN-FF methods were used for geometry optimization $[78,79,80,81]$. The $\mathrm{M}_{2} \mathrm{~L}_{4}$ cage geometries were optimized using GFN2-xTB and GFN-FF. The GBSA solvation method as implemented in XTB was used with THF as solvent for most optimizations to implicitly account for solvent effects [82, 83]. Thermochemical parameters such as the Gibbs free energy were computed using the hessian matrix calculations. These GFNn ( $n=2$, FF) methods are denoted as GFNn-xTB(THF) or GFNn-xTB(GAS) depending on whether GBSA solvation was used.

\subsection{Density Functional Theory}

\subsubsection{Pincer complexes}

Gaussian 16 C.01 was used to perform DFT calculations [84]. The BP86 exchange-correlation functional was used for geometry optimizations together with the def2SVP basis set $[85,86]$. This combination of functional and basis set have shown reliable geometry predictions accompanied with low computational costs $[87,88]$. Geometry optimizations were performed in the gas phase. Hessian calculations were performed for these geometries to verify the absence of imaginary frequencies and that each geometry corresponded to a local minimum on its respective potential energy surface (PES). Thermochemical parameters such as the Gibbs free energy were computed using the gas phase hessian calculations. Single point (SP) DFT calculations were performed on the gas-phase optimized geometries using the SMD solvation (THF) model [89]. SP calculations were performed using BP86 or PBE1PBE (also known as PBE0) functional with the def2TZVP basis set to further refine the obtained (free) energies and other thermochemical/electronic properties [86, 90]. All DFT calculations were performed with Grimme's D3 dispersion corrections [91]. These composite methods (geometry optimziation followed by SP), BP86/def2-SVP//XC/def2-TZVP (THF), are denoted as XC(THF) or XC(GAS) depending on the exchange-correlation $(\mathrm{XC})$ functional used. All geometries were pre-optimized with a combination of Openbabel's GAFF and UFF methods and/or GFN2-xTB before being subjected to full DFT based optimization. We did not conduct any conformational search.

The catalysts are denoted as $M-L$ where $M$ represents the metal center and $L$ the ligand. Reactive adosprtion of a $\mathrm{H}-\mathrm{X}$ species $(\mathrm{X}=\mathrm{H}, \mathrm{Br}, \mathrm{OH}, i-\mathrm{PrO})$ over $\mathrm{M}-\mathrm{L}$ leads to the formation of $M(X)-L(H)$ species. The thermodynamic stability of the $M(X)-L(H)$ was estimated by computing the Gibbs free energy and total energy change under standard conditions upon addition of the 
$\mathrm{H}-\mathrm{X}$ moiety.

$$
\begin{array}{r}
\mathrm{H}-\mathrm{X}+\mathrm{M}-\mathrm{L} \longrightarrow \mathrm{M}(\mathrm{X})-\mathrm{L}(\mathrm{H}) \\
\Delta G_{\mathrm{HX}}^{\circ}=G(M(X)-L(H))-G(M-L)-G(\mathrm{H}-\mathrm{X}) \\
\Delta E_{\mathrm{HX}}^{\circ}=E(M(X)-L(H))-E(M-L)-E(\mathrm{H}-\mathrm{X})
\end{array}
$$

\subsubsection{Co porphyrins}

TeraChem v1.94V-2019.08-beta was used to perform GPU-accelerated DFT SP calculations using the PBE1PBE XC and LANL2DZ basis sets with an effective core potential (ECP) on selected Co-Porphyrin geometries optimized using the GFN2-xTB method [92, 93, 94, 95].

\subsection{Root-mean-square deviation of atomic positions (RMSD)}

The RMSD is used to compare two molecular structures. In this approach, the minimal difference between the positions of the same atom on both molecular structures is used. The RMSDs were calculated using a Python program which uses the Kabsch or Quaternion algorithm to align the two molecular structures [96, 97, 98]. If for example the two molecules $\mathbf{p}$ and $\mathbf{q}$ with $\mathrm{n}$ points (atoms) are compared, the RMSD is defined as:

$$
\begin{aligned}
\operatorname{RMSD}(\mathbf{p}, \mathbf{q}) & =\sqrt{\frac{1}{n} \sum_{i=1}^{n}\left\|p_{i}-q_{i}\right\|^{2}} \\
& =\sqrt{\frac{1}{n} \sum_{i=1}^{n}\left(\left(p_{i x}-q_{i x}\right)^{2}+\left(p_{i y}-q_{i y}\right)^{2}+\left(p_{i z}-q_{i z}\right)^{2}\right)}
\end{aligned}
$$

\section{Code implementation}

An overview of the workflow of ChemSpaX is shown in Figure 2. The user has to supply: a molecule that needs to be functionalized (skeleton), which sites on the skeleton should be functionalized (functionalization_list) and what substituent should be placed on the supplied site (substituent). Substituents can be chosen from a pre-made database shipped with ChemSpaX or users can create new substituents in $X Y Z$ or MDL Molfile format [99]. Information for the correct placement of a substituent is kept in a CSV file. The CSV file stores: 1) Coordinates of the central atom of the substituent group which coordinates directly to the sites defined in the functionalization_list. 2) Coordinates of the centroid vector. A centroid vector, for a tetrahedral substituent such as $\mathrm{CH}_{3}$ is the vector connecting the central atom (C) to the centroid of the triangle formed by the three edges (hydrogens). for a planar substituent such as $\mathrm{NH}_{2}$ the centroid vector connects the center of the line joining the two hydrogen atoms to $\mathrm{N}$ (central atoms).

The substituent group is first correctly oriented using rotation matrices and placed at a pre-defined distance from the substitution site. Next GAFF followed by UFF optimization methods from Open Babel are used to selectively optimize the newly placed substituent via a constrained optimization protocol while keeping the original molecular skeleton frozen $[73,74]$. This combination of GAFF and UFF was found by trial-and-error (see SI). The correction in orientation of the substituent group prior to FF optimizations is needed to ensure a reasonable input geometry. The FF optimizations are based on MDL molfiles which contain the connectivity information between different atoms. When new substituents are placed the 
connectivity information is carefully updated to ensure that no undesired overlaps/connections are present. FF optimization using the MDL molfiles as input rectifies the geometry and removes any physical overlap between different atoms/functional groups. It is recommended to use a DFT optimized geometry as input skeleton since the FF optimization only influences the newly placed functional group. This choice helps keep the core of the geometry as close to its DFT optimized input structure as possible upon serial functionalization, while preventing steric hindrance from newly placed substituents cheaply via force-field optimizations. 

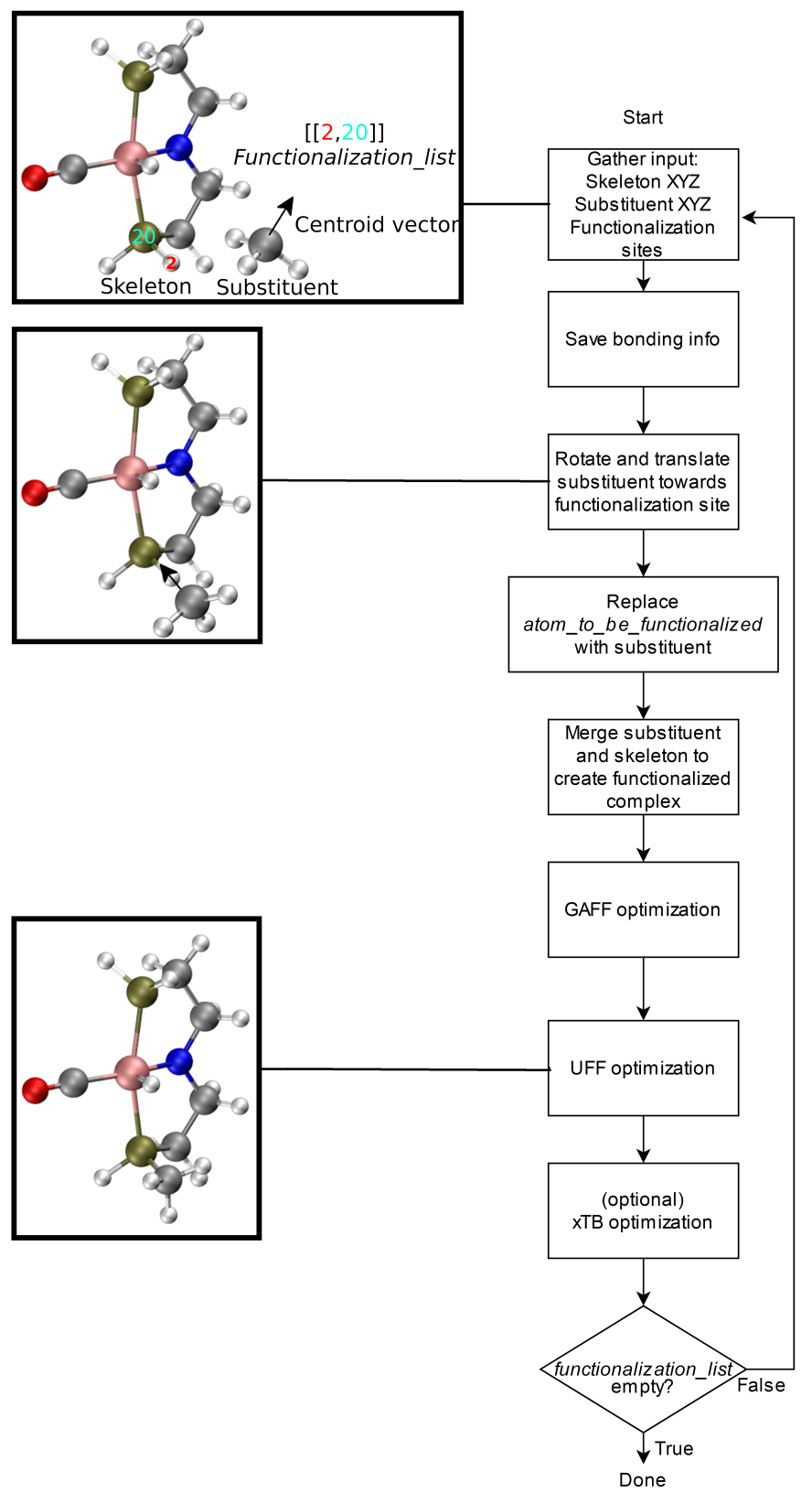

Figure 2: Overall workflow of ChemSpaX. 1) The user supplies the $X Y Z$ coordinates of a molecular skeleton, functionalization_list, and substituents. 2) The $X Y Z$ files are converted to MDL Molfiles to conserve correct bonding info. 3) The central atom of the substituent group and the centroid vector are used to rotate and translate the substituent group towards the functionalization site. 4) atom_to_be_functionalized is replaced by the substituent group. 5) The skeleton and substituent group are merged in one MDL Molfile with correct bonding information from input MDL Molfiles. 6) GAFF optimization is done to prevent steric hindrance. 7) Additionally, UFF optimization is done to prevent GAFF related issues. 8) Optionally, xTB optimization can be used for further optimization of the functionalized skeleton. 9) If there are no functionalizations left to do, the program exits and the functionalized skeleton is saved in MDL Molfile format. Else the functionalized skeleton will be used as input and the entire process repeats from step 1. 


\section{Results and discussion}

\subsection{Pincer complexes}

In this section the functionalization of the ligand scaffold of $\mathrm{Ru}$ and $\mathrm{Mn}$ based pincer complexes is described. In section 4.1.1 the functionalization strategy for both types of pincer complexes is shown. Subsequently, the quality of geometries generated by ChemSpaX is compared to higher level methods in section 4.1.2. An investigation of thermochemical properties is done in section 4.1.3.

\subsubsection{Functionalization strategy}

The functionalization strategy for the RuPNP complex is shown in Figure 3. This functionalization strategy resulted in $144(\mathrm{M}-\mathrm{L})$ complexes. For each $\mathrm{M}-\mathrm{L}$ complex, its hydrogenated version $M(H)-L(H)$ was also generated by functionalization of the $M(H)-L(H)$ complex as skeleton, leading to a total of 288 geometries. Out of these, 27 pairs $(M-L / M(H)-L(H))$ were selected for BP86(GAS) optimization. BP86(THF) and PBE1PBE(THF) PS calculations were performed on the DFT optimized geometries.

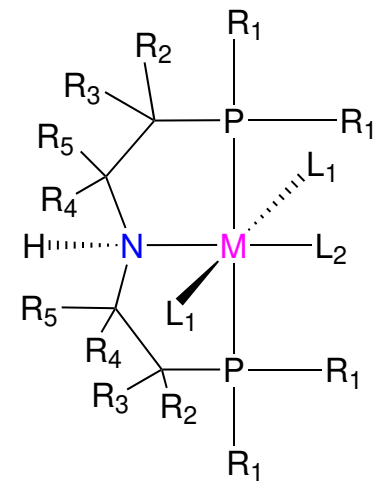

$$
\begin{aligned}
& \mathrm{R}_{\mathrm{i}}=\left\{\mathrm{Me}, \mathrm{Et}, i-\mathrm{Pr}, \mathrm{Ph}, \mathrm{CF}_{3}\right\} ; \mathrm{L}_{1}=\mathrm{H} \\
& \mathrm{L}_{2}=\left\{\mathrm{CO}, \mathrm{PMe}_{3}\right\} ; \mathrm{M}=\mathrm{Ru}
\end{aligned}
$$

Figure 3: Functionalization strategy for the RuPNP pincer complexes.

Krieger and co-workers [63] used an "under development" version of ChemSpaX to generate a database of $1225 \mathrm{Mn}$ complexes based on five representative ligand scaffolds namely PNP-(bis(3-phosphaneylpropyl)amine)- ${ }^{1}$, SNS(azanediylbis(ethane-1-thiol)), CNC(bis(2(1H-3 $\lambda^{4}$-imidazol-3-yl)ethyl)amine), PNN( $\mathrm{N}^{1}$-(2-phosphaneylethyl)ethane-1,2-diamine), and PCP $\left(\mathrm{N}^{1}, \mathrm{~N}^{3}\right.$-bis(phosphaneyl)benzene-1,3-diamine) $[100,101,102,103,104,105,106]$. Out of these 1225 geometries, 545 geometries containing the PCP, PNN and CNC ligand backbones were selected for DFT optimization using BP86(GAS). This dataset of 545 complexes will be discussed further.

The functionalization strategy for PCP, PNN and CNC ligand based complexes is show in Figure 4. Functionalizations were performed symmetrically keeping all four $\mathrm{R}_{1}$ sites the same. Similarly both $R_{2}$ sites (only 1 in case of PCP backbone) were functionalized with the same groups. However, $R_{1}$ and $R_{2}$ were not constrained to be the same.

\footnotetext{
${ }^{1}$ This is not the same PNP ligand as used for the Ru complexes. The PNP ligand used for Mn complexes contains a propyl bridge while the one used for Ru complexes contains an ethyl bridge
} 


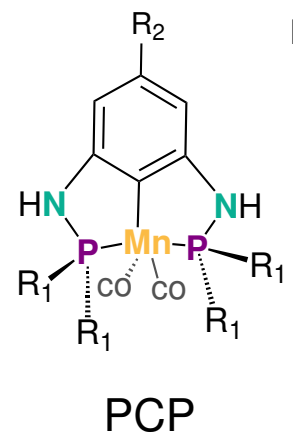

$\mathrm{R}_{\mathrm{i}}=\left\{\mathrm{CF}_{3}, \mathrm{H}, \mathrm{Ph}, i-\mathrm{Pr}, \mathrm{cy}, t-\mathrm{Bu}\right\}$

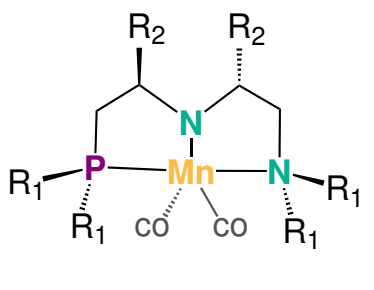

PNN

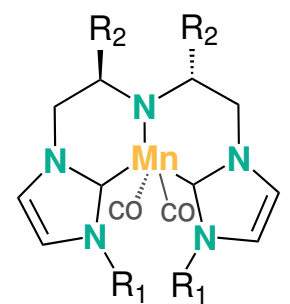

CNC

Figure 4: Functionalization strategy for $M n$-pincers with various donor $\left(R_{1}\right)$ and backbone $\left(R_{2}\right)$ groups.

\subsubsection{Quality assessment of generated geometries}

ChemSpaX uses FF optimizations on newly placed substituent groups in each iteration. Therefore we name geometries generated directly by ChemSpaX as "FF-geometries". To assess the quality of FF-geometries they were compared against GFN2-xTB and DFT. The quality of geometries was assessed along two dimensions: energy and spatial. When comparing geometries along the energy dimension, we computed the net energy change of a particular chemical reaction using SP calculations on FF-geometries at higher levels of theory (e.g. DFT or GFN2-xTB). For a given reaction the ChemSpaX generated FF geometries of reactants and product were taken. Each FF geometry underwent two calculations: 1) a SP calculations where the energy of the FF geometry was evaluated at DFT level of theory and the electronic energy change of the reaction $\Delta \mathrm{E}_{\mathrm{FF} / / \mathrm{DFT}-\mathrm{SP}}$ was calculated 2) a full DFT based optimization was carried using the FF geometry as input resulting in a new geometry and energy at the DFT level of theory. The corresponding electronic energy change of the reaction $\Delta \mathrm{E}_{\mathrm{DFT}}$ was calculated. The difference between the reaction energies is computed using $\Delta \mathrm{E}_{\mathrm{FF} / / \mathrm{DFT}-\mathrm{SP}}$ and $\Delta \mathrm{E}_{\mathrm{DFT}}$ to get $\Delta \Delta \mathrm{E}_{\mathrm{FF}}$ which is a metric for the quality of FF geometry for the reaction being investigated (Equation 6). A similar approach was used by Sinha and co-workers to assess the quality of GFN2-xTB optimized geometries against DFT (Equation 7) [107].

$$
\begin{gathered}
\Delta \Delta E_{F F}=\Delta E_{D F T}-\Delta E_{F F / / D F T-S P} \\
\Delta \Delta E_{G F N 2-x T B}=\Delta E_{D F T}-\Delta E_{G F N 2-x T B / / D F T-S P}
\end{gathered}
$$

Such a comparison allows us to estimate the range of error caused by the direct use of FFgeometries by skipping computationally expensive DFT optimizations, for example in highthroughput screening (HTS) workflows. Geometries were also assessed along the spatial dimension where we computed the RMSD between FF-geometries against GFN2-xTB, and DFT optimized geometries.

For RuPNP complexes we chose the hydrogenation reaction $\left(\mathrm{M}-\mathrm{L}+\mathrm{H}_{2} \longrightarrow \mathrm{M}(\mathrm{H})-\mathrm{L}(\mathrm{H})\right.$ ) to investigate $\Delta \Delta \mathrm{E}_{\mathrm{FF}}$ and $\Delta \Delta \mathrm{E}_{\mathrm{GFN} 2-\mathrm{xTB}}$. The corresponding mean $(\mu)$ and standard deviation $\left(\sigma^{2}\right)$ were also computed ${ }^{2}$. We found $\mu\left(\Delta \Delta E_{F F}\right)=7.20 \mathrm{kcal} \mathrm{mol}^{-1}$ with $\sigma^{2}\left(\Delta \Delta E_{F F}\right)$ $=4.57 \mathrm{kcal} \mathrm{mol}^{-1}$; and $\mu\left(\Delta \Delta E_{G F N 2-x T B}\right)=4.77 \mathrm{kcal} \mathrm{mol}^{-1}$ and $\sigma^{2}\left(\Delta \Delta E_{G F N 2-x T B}\right)=$

\footnotetext{
${ }^{2} \Delta \Delta \mathrm{E}_{\mathrm{GFN} 2-\mathrm{xTB}}: 20$ pairs; $\left(\Delta \Delta \mathrm{E}_{\mathrm{FF}}\right) 25$ pairs $\mathrm{M}-\mathrm{L} / \mathrm{M}(\mathrm{H})-\mathrm{L}(\mathrm{H})$ geometries
} 
$2.57 \mathrm{kcal} \mathrm{mol}^{-1}$. This indicates an overall good agreement between the GFN2-xTB optimized structures and the structures generated by ChemSpaX.

For the Mn-pincer complexes we investigated reactive adsorption of $\mathrm{H}-\mathrm{X}$ species $(\mathrm{M}-\mathrm{L}+$ $\mathrm{H}-\mathrm{X} \longrightarrow \mathrm{M}(\mathrm{X})-\mathrm{L}(\mathrm{H}) ; \mathrm{X}=\mathrm{H}, \mathrm{Br}, i-\mathrm{PrO}$, and $\mathrm{OH}$ ) with the reaction energetics characterized by $\Delta \mathrm{E}$, and the respective $\mathrm{FF}$ geometries by $\Delta \Delta \mathrm{E}_{\mathrm{FF}}$ respectively. When the entire dataset was considered i.e. all three ligand systems, and all four $\mathrm{H}-\mathrm{X}$ species, we found $\mu=$ $25.57 \mathrm{kcal} \mathrm{mol}^{-1}$ and $\sigma^{2}$ of $26.08 \mathrm{kcal} \mathrm{mol}^{-1}$. We also investigated the mean and standard deviation values associates with each ligand and $\mathrm{H}-\mathrm{X}$ species (Table 1 ). FF geometries associated with the PCP ligand were found to have the lowest $\mu$ and $\sigma^{2}$ values in general, followed by the CNC ligand complexes. The PNN ligand complexes had the highest $\mu$ and $\sigma^{2}$. In terms of the $\mathrm{H}-\mathrm{X}$ species considered, $\Delta \Delta \mathrm{E}_{\mathrm{FF}}$ computed for both $\mathrm{HBr}$ and $\mathrm{H}_{2}$ showed lower $\mu$ while those for $i$ - $\mathrm{PrOH}$ and $\mathrm{H}_{2} \mathrm{O}$ showed highest $\mu$. The reason for worse performance of $i$ - $\mathrm{PrOH}$ and $\mathrm{H}_{2} \mathrm{O}$ can be attributed to the observed recombination of $\mathrm{O}-\mathrm{H}$ bond in many geometries during DFT based optimization leading to $\mathrm{M}(\mathrm{H}-\mathrm{X})-\mathrm{L}$ type complexes, or non-adsorbed $\mathrm{H}-\mathrm{X}$ adducts.

Table 1: $\mu \pm \sigma^{2}$ for $\Delta \Delta \mathrm{E}_{\mathrm{FF}}$ for various $\mathrm{H}-\mathrm{X}$ and ligands in $\mathrm{kcal}^{\mathrm{mol}}{ }^{-1}$.

\begin{tabular}{|c||c|c|c|c|}
\hline Ligand & $\mathrm{Br}$ & $\mathrm{H}$ & $\mathrm{OH}$ & $i$-PrO \\
\hline \hline $\mathrm{PCP}$ & $17.10 \pm 4.76$ & $10.80 \pm 7.25$ & $20.96 \pm 9.36$ & $25.60 \pm 23.45$ \\
\hline PNN & $36.37 \pm 16.83$ & $28.05 \pm 18.42$ & $63.28 \pm 36.79$ & $56.06 \pm 32.05$ \\
\hline CNC & $8.94 \pm 8.08$ & $12.16 \pm 9.64$ & $8.31 \pm 10.97$ & $16.92 \pm 22.83$ \\
\hline
\end{tabular}

The distribution of $\Delta \Delta \mathrm{E}_{\mathrm{FF}}$ for the reactive adsorption of $\mathrm{HBr}$ for all the ligands and functionalization sites $\left(R_{1}\right.$ and $\left.R_{2}\right)$ is shown in Figure 5 . For the CNC ligand complexes variation of the functional group at the $\mathrm{R}_{2}$ position with fixed $\mathrm{R}_{1}$ (Figure $5 \mathrm{a}$ )) led to similar $\mu\left(\Delta \Delta \mathrm{E}_{\mathrm{FF}}\right)<10$ kcal mol ${ }^{-1}$ for all groups except for $\mathrm{R}_{1}=\mathrm{CF}_{3}$. Since functionalization site $\mathrm{R}_{2}$ is primarily a ligand backbone site, it seems that the effective relaxation of the ligand during DFT optimization is similar for the CNC complexes. Variation of the functionalization group at $\mathrm{R}_{1}$ results in a wider spread in the $\Delta \Delta \mathrm{E}_{\mathrm{FF}}$ values for $\mathrm{CF}_{3}, \mathrm{H}$ and cy groups, while $i$-Pr substituents show most narrow distribution with $\mu\left(\Delta \Delta \mathrm{E}_{\mathrm{FF}}\right)=9.01 \mathrm{kcal} \mathrm{mol}^{-1}$. The difference in distribution spread probably arises from the worse performance of force-field methods for geometric fragments close to the metal center leading to higher structure-energy sensitivity. All PNN ligand complexes showed high and widespread $\mu\left(\Delta \Delta \mathrm{E}_{\mathrm{FF}}\right)$ values. Most notable is $\mathrm{R}_{1}=\mathrm{H}$ which shows a rather large spread indicating large geometric relaxations when different ligands are introduced on the backbone sites. PCP ligand complexes are interesting for their almost linearly increasing $\mu\left(\Delta \Delta \mathrm{E}_{\mathrm{FF}}\right)$ as the size of the functional group on the $\mathrm{R}_{1}$ site is increased while the spread in energies is minimal (Figure $5 \mathbf{e})$ ). Furthermore, the variation at site $\mathrm{R}_{2}$ shows an almost constant $\mu\left(\Delta \Delta \mathrm{E}_{\mathrm{FF}}\right)$ with similar spread ( Figure $\mathbf{5} \mathbf{f}$ )). This observation indicates the source of spread is related to a larger geometric relaxation near the metal site during DFT based geometry optimization. Site $\mathrm{R}_{2}$ being farther away from the metal center seems to have minimal impact on the geometries and is rather well optimized by the force-field. This observation is consistent with the finding that variation of $R_{2}$ had minimal impact on thermochemical properties in Mn-PCP complexes [63]. We also compared the individual difference in energy between FF and DFT optimized geometries for $M(X)-L(H)$ and $M-L$ complexes. In general, we found that the energy of FF optimized $\mathrm{M}(\mathrm{Br})-\mathrm{L}(\mathrm{H})$ complexes were closer to their DFT counterparts when compared to corresponding $M-L$ complexes. This observation reveals that the $M-L$ complexes have a larger contribution to the $\Delta \Delta \mathrm{E}_{\mathrm{FF}}$. We also observed that almost all cases where the $\mathrm{M}(\mathrm{X})-\mathrm{L}(\mathrm{H})$ complexes had a larger contribution to the $\Delta \Delta \mathrm{E}_{\mathrm{FF}}$ were based on the CNC ligand (24 entries) with $\mu=9.4 \mathrm{kcal}$ 


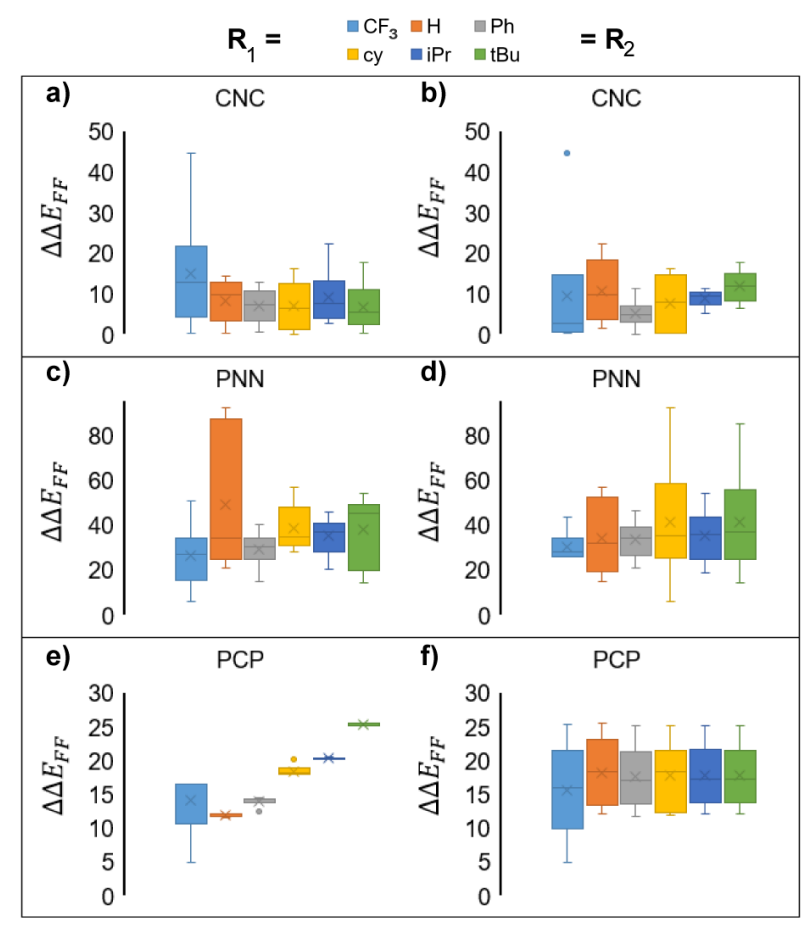

Figure 5: Box plots showing the distribution of $\Delta \Delta \mathrm{E}_{\mathrm{FF}}$ for reactive adsoprtion of $\mathrm{H}-\mathrm{X}(\mathrm{X}=$ $\mathrm{Br})$ for $(\mathrm{a}, \mathrm{b}) \mathrm{CNC}(\mathrm{c}, \mathrm{d})$ PNN and (d,e) PCP ligand based Mn complexes. For each ligand functionalization effect of the donor $\left(R_{1}\right)$ and backbone $\left(R_{2}\right)$ groups on the spread of $\Delta \Delta E_{F F}$ is visualized.

$\mathrm{mol}^{-1}$.

The quality of FF geometries along the spatial dimension was analyzed via RMSD between geometries produced by DFT or GFN2-xTB based optimizations. Specifically we computed the cartesian heavy-atom (all elements except $\mathrm{H}$ ) root-mean-square deviation (hRMSD) [77]. Both FF $\left(\mu=0.67 \AA ; \sigma^{2}=0.30 \AA\right)$ and GFN2-xTB $\left(\mu=0.41 \AA ; \sigma^{2}=0.34 \AA\right)$ structures had a similar average hRMSD when compared to DFT structures. Distributions of the hRMSD values are shown in the SI. A selection of the geometries are visualized using structure overlay plots in Figure 6. The comparisons in these structure overlay plots is done as follows: a) the FF optimized structure (silver), generated by ChemSpaX, is compared to a DFT optimized structure (green) and b) a GFN2-xTB optimized structure (silver) is compared to a DFT optimized structure (green).

A comparison using the hRMSD was done in a similar manner for the $\mathrm{Mn}$-pincer complexes. It was again observed that both FF $\left(\mu=0.70 \AA ; \sigma^{2}=0.45 \AA\right)$ and GFN2-xTB $\left(\mu=0.78 \AA ; \sigma^{2}\right.$ $=0.67 \AA$ ) structures had a similar average hRMSD when compared to DFT structures, albeit with moderately high standard deviations. This is consistent with the previous observation from the RuPNP structures, that the structures generated by ChemSpaX are in good agreement with GFN2-xTB optimized structures. It should be noted here that the GFN2-xTB optimizations were performed in the solvated phase (GBSA(THF)) while the DFT optimizations are in the gas phase. 


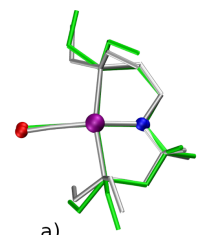

hRMSD $=0.44$ $R_{1}=E t, R_{4}=R_{5}=M e$

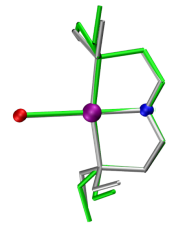

$$
\text { a) }
$$

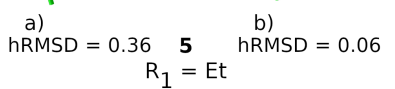

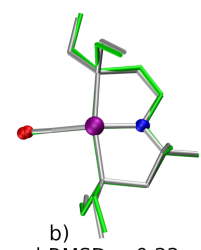

b) 1

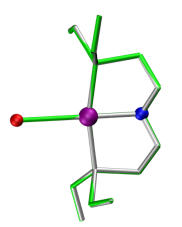

$\mathrm{R}_{1}=\mathrm{E}$

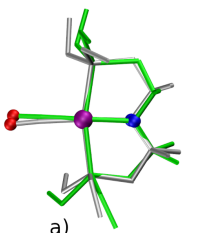

hRMSD $=0.67$

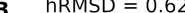

$\mathrm{R}_{1}=\mathrm{Et}, \mathrm{R}_{4}=\mathrm{R}_{5}=\mathrm{Me}$

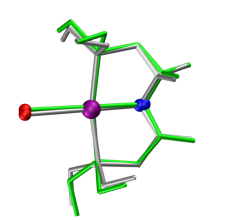

a)

SRD $=0.30 \quad 3-\mathbf{H} \quad$ hRMSD $=0.08$

$\mathrm{R}_{1}=\mathrm{Et}, \mathrm{R}_{4}=\mathrm{R}_{5}=\mathrm{Me}$
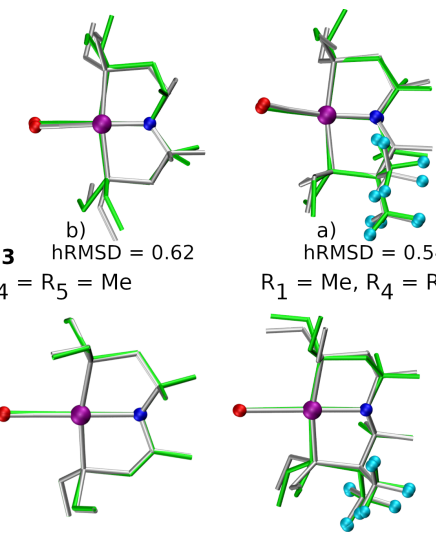

a)

hRMSD $=0.33 \quad$ b) $R_{1}=M e, R_{4}=R_{5}=M e, R_{2}=R_{3}=C F_{3}$

Figure 6: Structure overlay plots of some selected TM complexes. a) shows FF optimized (silver) vs DFT optimized (green) structures and b) shows GFN2-xTB optimized (silver) vs DFT optimized (green) structures. The '-H' indicates that the complex is hydrogenated. Color code used for elements: red $=\mathrm{O}$, purple $=\mathrm{Ru}$, dark-blue $=\mathrm{N}$ and turquoise $=\mathrm{F}$.

The hRMSD analysis was performed for various ligands backbones. This comparison is shown in Figure 7. As observed by Krieger and co-workers, DFT and xTB based optimization of most PNN based complexes resulted in hemilabile ligands [63]. Due to this hemilability, the spread of the PNN backbone hRMSD data was larger compared to the other backbones. For the PNN and CNC backbones it was observed that functionalization with electron donating substituents on the $\mathrm{R}_{1}$ site resulted in a higher hRMSD. For the PCP backbone it was observed that functionalization with $t$-Bu on the $\mathrm{R}_{1}$ site specifically gave a larger hRMSD. This observation is expected to have the following underlying causes: 1) electron donating groups like $t$-Bu are bulkier, have more number of atoms and a complex structure which increases the chance of accumulating an error (vide infra) 2) electron donating groups affect the electronic density in the entire complex and may cause and may elicit shearing of the skeleton which is kept frozen in FF calculations. When optimized with DFT, the skeleton would relax and this would lead to a higher hRMSD.

We that $\Delta \Delta \mathrm{E}_{\mathrm{FF}}(\mathrm{HBr})$ and $\Delta \Delta \mathrm{E}_{\mathrm{FF}}\left(\mathrm{H}_{2}\right)$ correlated well (Pearson correlation coefficient $(\mathrm{R})$ $=+0.87$ ) opening up possibilities to reduce computational effort in screening through these intermediates. Krieger and co-workers had reported a similar albeit stronger correlation $(\mathrm{R}=$ 0.95) between $\Delta \mathrm{G}_{\mathrm{HBr}}$ and $\Delta \mathrm{G}_{\mathrm{H}_{2}}$ [63]. 


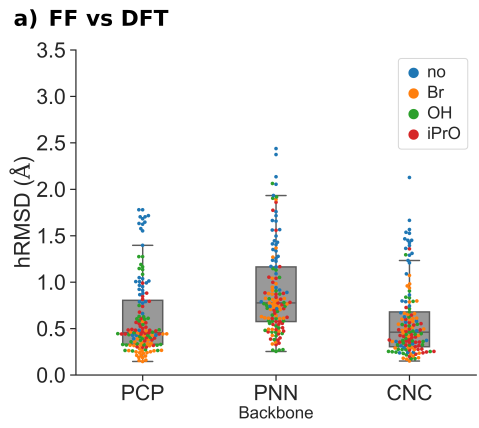

b) GFN2-xTB vs DFT

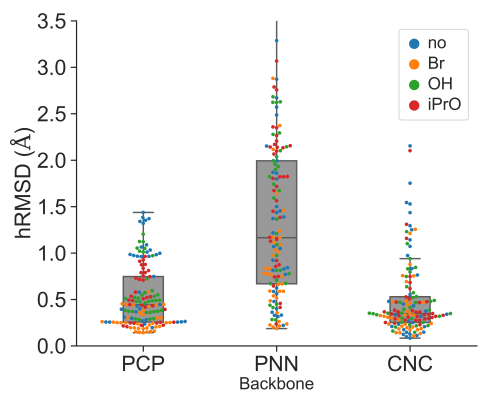

Figure 7: Comparison of hRMSD for the PCP, PNN and CNC ligand backbone of a) ChemSpaX generated FF structures compared to DFT optimized structures, b) GFN2-XTB optimized structures compared to DFT optimized structures. The various adducts bonded to the metal center are color coded, where 'no' means that no adduct is bonded to the metal center. All energy values are in $\mathrm{kcal} \mathrm{mol}^{-1}$.

\subsubsection{Comparison of $\Delta G$ and $\Delta E$}

Calculation of the hessian matrices of the geometry of reactant and product species is often needed to estimate the Gibbs free energy $\Delta \mathrm{G}$ change in a chemical reaction. Hessian calculation is also quintessential to ensure accurate and reliable geometry optimization. However, it would be desirable to omit these computationally expensive calculations when filtering through a large number of molecular structures for example in a HTS workflow. Sinha and co-workers had reported a strong correlation between $\Delta \mathrm{G}$ with $\Delta \mathrm{E}$ for the proton transfer reaction [107]. In the present work we found a similar correlation to hold for the reactive adsorption of $\mathrm{H}$-X species over Ru-PNP $(\mathrm{X}=\mathrm{H})$ and $\mathrm{Mn}$-pincers $(\mathrm{X}=\mathrm{H}, \mathrm{Br}, i-\mathrm{PrO}, \mathrm{OH})$ (Figure 8). This correlation shows that $\Delta \mathrm{E}$ scales linearly with $\Delta \mathrm{G}$. The linear fit in Figure 8 shows that the difference in $\Delta \mathrm{E}$ and $\Delta \mathrm{G}$ is approximately constant and equals $15 \mathrm{kcal} \mathrm{mol}^{-1}$. Therefore, $\Delta \mathrm{E}$ can be used instead of $\Delta \mathrm{G}$ with a minimal loss of accuracy, avoiding computationally expensive hessian calculations in HTS applications (RMSE(Ru-PNP) $=1.7 \mathrm{kcal} \mathrm{mol}^{-1}$; RMSE(Mn-pincers) $=1.9$ $\mathrm{kcal} \mathrm{mol}^{-1}$ ). We would like to emphasize here that when accurate results are really needed hessian calculations must be performed to ensure that the optimized geometry is a minimum on the potential energy surface. 

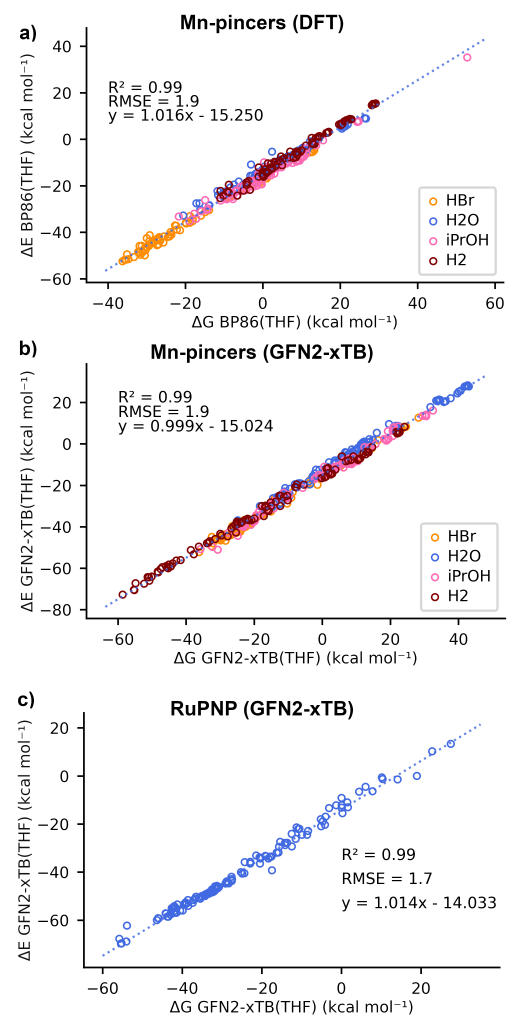

Figure 8: Comparison between the net Gibbs free energy $(\Delta \mathrm{G})$, and the net electronic energy $(\Delta \mathrm{E})$ for the reaction $\mathrm{M}-\mathrm{L}+\mathrm{H}-\mathrm{X} \longrightarrow \mathrm{M}(\mathrm{X})-\mathrm{L}(\mathrm{H})$ computed via a) DFT calculations for $\mathrm{Mn}-$ pincers, b) GFN2-xTB calculations for Mn-pincers and c) GFN2-xTB calculations for RuPNP [107]. Note that for plot b) data for $\mathrm{H}-\mathrm{X}=t-\mathrm{BuOH}, \mathrm{EtOH}$, and $\mathrm{MeOH}$ computed in the reference [63] was also included.

\subsection{Co-porphyrin}

Porphyrins are widely investigated, for example, for their applications in biocatalysis, organic photovoltaics, molecular wires and many more applications [108, 109, 110, 111]. This wide variety of applications has been enabled by the design and synthesis of structurally diverse porphyrins [108]. Postfunctionalization is a common method to tune the electronic and chemical properties of porphyrins, where functional groups and substituents are introduced after the construction of the porphyrin macrocycle. However, experimental exploration of the chemical space of porphyrins is limited by synthetic and economic feasibility. This is where computer-aided molecular design tools can be helpful.

Apart from the chemical application perspective, investigating the functionalization of porphyrins is also of use for further refinement of our workflow. When functionalizing a structure as implemented in ChemSpaX (freezing the skeleton and performing FF optimization only on the newly placed substituents), errors can be introduced. Stretching or compression of the skeleton structure is not taken into account since the skeleton is frozen. By investigating a structure that is close to $2 \mathrm{D}$ instead of $3 \mathrm{D}$, like porphyrins, the assessment of the introduced errors and their propagation through the workflow of ChemSpaX is simplified. 
We performed functionalization of Co porphyrins following a serial functionalization strategy ${ }^{3}$ to create a database of $\sim 1100$ complexes. Section 4.2.1 discusses the functionalization strategy. Subsequently, the propagation of errors introduced in geometries generated by ChemSpaX is investigated in section 4.2.2. An investigation of the HOMO-LUMO gap is done in section 4.2.3.

\subsubsection{Functionalization strategy}

Figure 9 shows the functionalization strategy for Co porphyrin. The Co porphyrin skeletons were functionalized with various phenyl groups on the $R_{1}$ sites to generate 10 new skeletons. These skeletons were then used to generate 1120 functionalized Co porphyrin complexes. The functionalization was done serially as described in Code implementation. The sites X1-X5 on the phenyl rings $\left(R_{1}\right)$ were functionalized first. Finally, functionalizations were done on $R_{2}$ and $\mathrm{R}_{3}$ respectively.

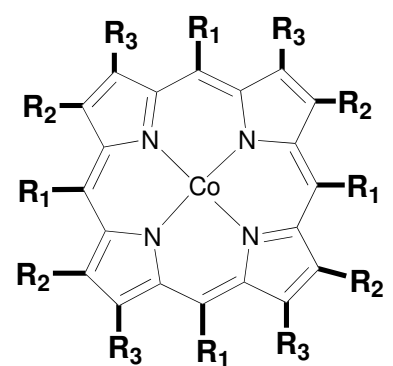<smiles>[Y]c1cc([X])c([Y4])c([Y4])c1[Y]</smiles>

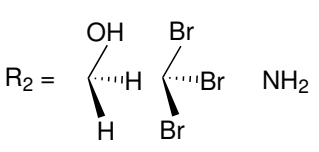<smiles>O=C(O)C(Cl)Cl</smiles>

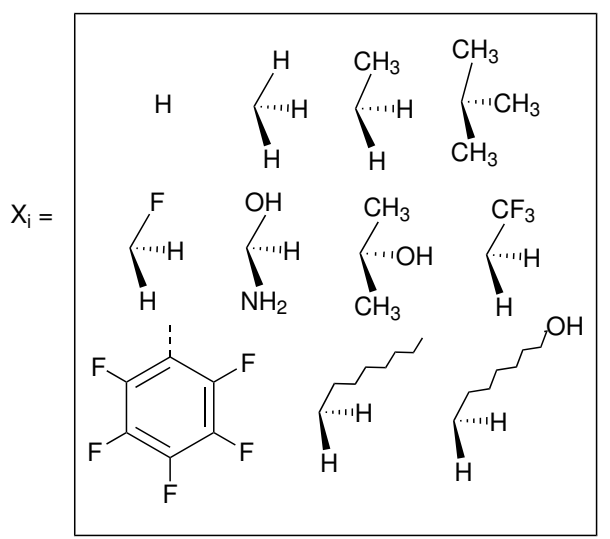

Figure 9: Functionalization strategy for Co porphyrin. Phenyl groups were first placed on the $R_{1}$ sites and subject to further functionalizations. With this strategy a database of 1120 Co porphyrin structures was generated.

The resulting complexes are shown for 3 different skeletons in Figure 10, where the skeleton, and Co-porphyrin complexes at the end of $5^{\text {th }}$, and $15^{\text {th }}$ functionalization are shown in a column demonstrating the geometric complexity introduced upon functionalization.

\footnotetext{
${ }^{3} \mathrm{~A}$ serial functionalization strategy means that the functional groups were placed one after another leading to more complex functionalized structures.
} 


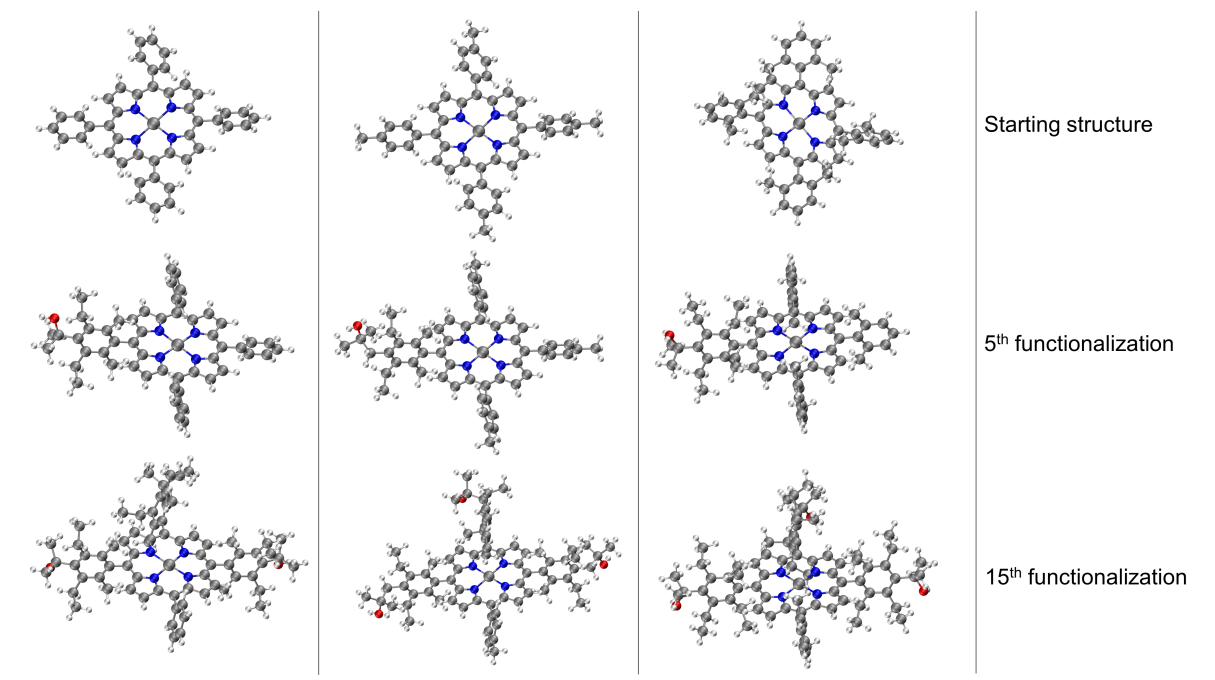

Figure 10: Functionalization strategy for Co porphyrin shown for 3 different skeletons. For each skeleton geometries resulting from the $5^{\text {th }}$, and the $15^{\text {th }}$ functionalization are shown in a column. The phenyl rings are functionalized symmetrically. In the $5^{\text {th }}$ functionalization the left most phenyl ring of the skeleton is functionalized, in the $10^{\text {th }}$ functionalization step the same substituents are placed on the upper phenyl ring, and on the right most phenyl ring in the $15^{\text {th }}$ step. Color code used for elements: gray $=\mathrm{C}$ (metal center $=\mathrm{Co})$, white $=\mathrm{H}$, red $=\mathrm{O}$, dark-blue $=\mathrm{N}$ and turquoise $=\mathrm{F}$.

\subsubsection{Error propagation of serial functionalization}

The 1120 geometries were optimized using GFN2-xTB(THF). The hRMSD between FF and GFN2-xTB optimized geometries was computed to compare the quality of the FF geometries generated by ChemSpaX, giving $\mu=1.28 \AA$, and $\sigma^{2}=0.54 \AA$. Upon detailed analysis of the hRMSD it was observed that the hRMSD increases nearly linear for each subsequent functionalization on a skeleton. The error introduced by placing a new substituent group is thus propagated upon the next placement of a substituent. An example is shown in Figure 11 where the hRMSD for each skeleton is plotted. The first 10 blocks of plots show the increasing hRMSD for each functionalization on a given skeleton. The last block shows the hRMSDs for all 10 skeletons, showing how the error increases almost linearly upon each functionalization regardless of the skeleton used for functionaliziation. These results could help users in devising an optimal strategy to get more accurate geometries at a balanced computational cost when using ChemSpaX. One can determine when an extra geometry optimization with a higher-level method, such as GFN2-xTB, is needed in-between functionalizations. The linear regression fits shown in Figure 11 can be used to estimate the hRMSD. One can generate these relations on a small sub-set of geometries in the functionalization scope by performing additional geometry optimizations at a higher level of theory. The predicted hRMSD can be used to set a threshold value. When this threshold is reached, a higher-level optimization method can be used to reduce the hRMSD and the functionalization can be continued with the optimized intermediate as a new skeleton. 

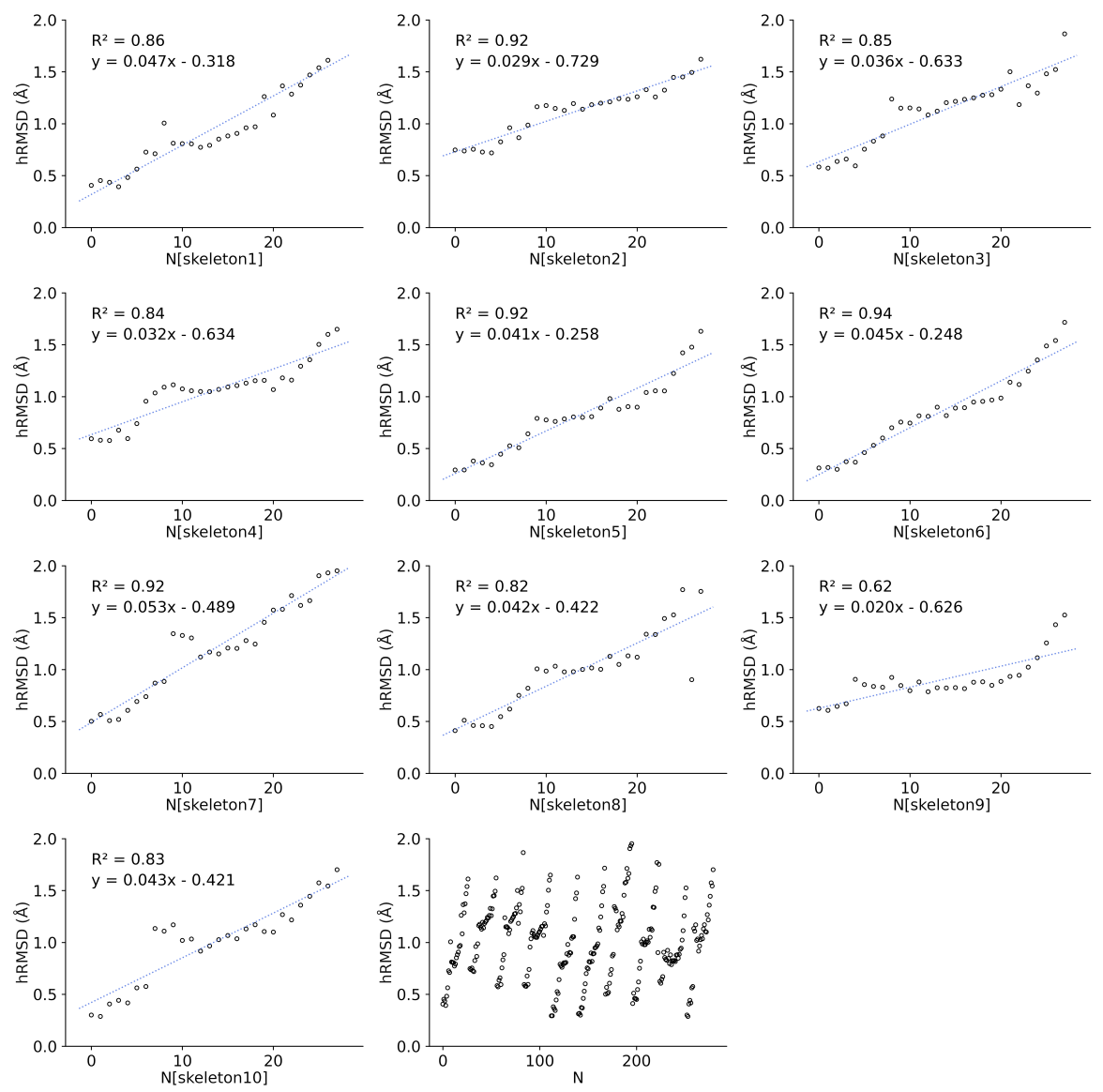

Figure 11: Increasing hRMSD for each functionalization on a given skeleton. Where $\mathrm{N}$ is the number of functionalizations, starting from 0.10 skeletons were created and 28 functionalizations were done for each skeleton. The first 10 blocks each represent a skeleton, while the last block on the bottom shows the increasing hRMSD for each skeleton grouped in 1 figure. After every $28^{\text {th }}$ functionalization $(0 \leq \mathrm{N} \leq 279)$, a new skeleton is functionalized.

Structure overlay plots of the FF geometries (silver) and the GFN2-xTB optimized geometries (green) are shown in Figure 12. The upper half of the figure shows structure overlay plots of complexes with $<200$ atoms while the lower half shows complexes with $>200$ atoms, and diverse hRMSDs. Large hRMSDs result from divergent orientation of long alkyl chain substituents, and fluorine containing groups 

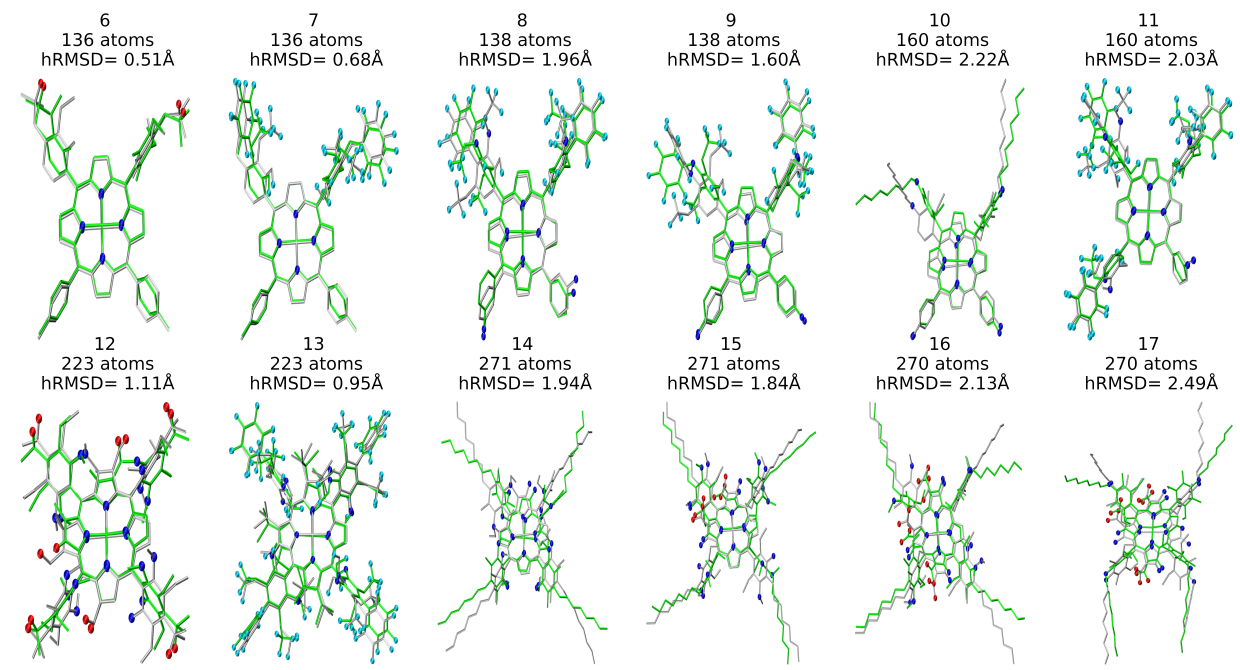

Figure 12: Structure overlay plots of selected Co porphyrin complexes. ChemSpaX generated (FF) structures (silver) are plotted against GFN2-xTB optimized (silver) structures. The upper half of the figure consists of structures with $<200$ atoms and the lower half of the figure shows structures $>200$ atoms. Color code used for elements: red $=\mathrm{O}$, dark-blue $=\mathrm{N}$ and turquoise $=\mathrm{F}$.

\subsubsection{HOMO-LUMO gap prediction}

Functional inorganic materials that are used for photocatalysis or photovoltaics require knowledge of the HOMO-LUMO gap, since this gap determines which wavelength of light is absorbed $[112,113,114]$. Additionally, the HOMO-LUMO gap can be used to predict the stability of TM complexes [115]. HOMO-LUMO gap prediction based on only the molecular structure as input using statistical methods, which avoid computationally expensive quantum chemistry calculations, can thus be a great resource to screen and develop functional inorganic materials [116].

We performed GPU accelerated DFT based SP calculations on GFN2-xTB optimized geometries of 280 porphyrin complexes out of the 1120 complexes generated using ChemSpaX. It was found that the hRMSD between FF geometries and GFN2-xTB optimized structures had a moderate correlation with the DFT (SP) computed HOMO-LUMO gap $(\mathrm{R}=-0.46)$ on the GFN2-xTB optimized geometry. This indicates that hRMSD could potentially be a feature in advanced machine learning applications for electronic property prediction. Additional analysis for prediction of the HOMO-LUMO gap can be found in the SI.

\section{3 $\mathrm{M}_{2} \mathrm{~L}_{4}$ cage}

The versatility of ChemSpaX is shown by the automated placement of substituents without introducing steric hindrance on a geometry that is more complex. An $\mathrm{M}_{2} \mathrm{~L}_{4}$ cage was functionalized at 16 sites with various substituent groups. The results are shown in figure Figure 13 . This serial functionalization yielded 16 structures and these were further optimized using GFN-FF and GFN2-xTB(GAS). The hRMSDs between FF geometries and those generated using the GFN-FF and GFN2-xTB optimization methods were calculated. The statistics shown for each optimization method in Table 2 revealed that the ChemSpaX generated FF geometries are 
closer to the GFN2-xTB optimized geometries compared to GFN-FF optimized geometries.
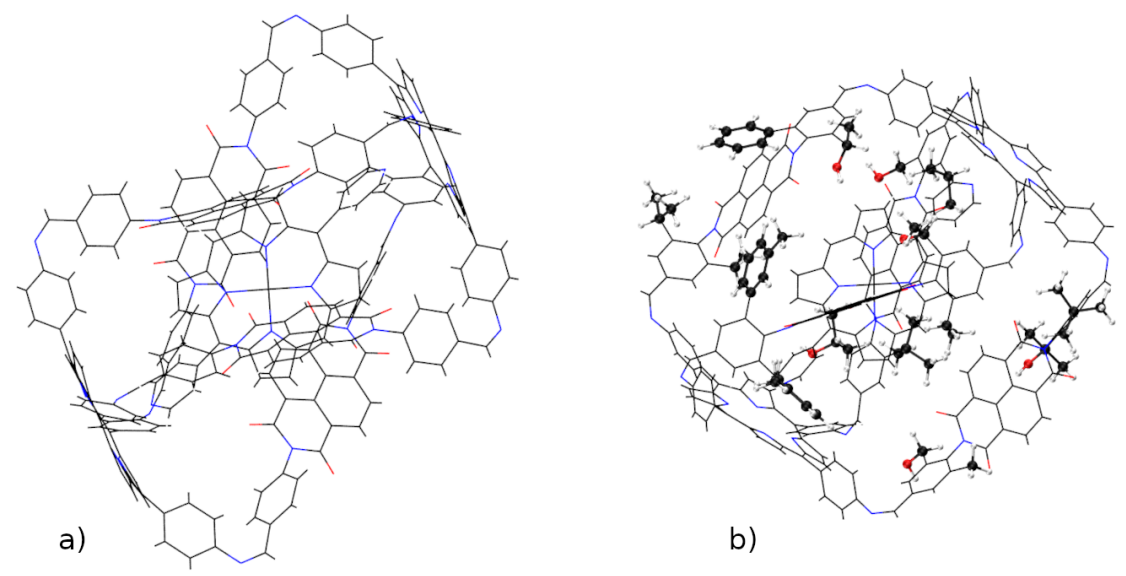

b)

Figure 13: A visualization of the functionalized $M_{2} L_{4}$ cage which shows a) the input skeleton and b) the GFN2-xTB optimized geometry after placement of 16 substituents. The newly placed substituents are shown in a distinguished representation.

Table 2: Statistics for the hRMSD between various methods. The two optimization methods that are compared to each other are shown in the first row.

\begin{tabular}{c|c|c|c}
\hline & GFN-FF v GFN2-xTB & FF v GFN2-xTB & FF v GFN-FF \\
\hline$\mu$ & $2.54 \AA$ & $2.14 \AA$ & $0.83 \AA$ \\
$\sigma^{2}$ & $0.34 \AA$ & $0.25 \AA$ & $0.26 \AA$ \\
Max. hRMSD & $3.18 \AA$ & $2.46 \AA$ & $1.37 \AA$ \\
\hline
\end{tabular}

\section{Summary and conclusions}

In this work, an automated Python-based workflow for the exploration of local chemical space is presented. ChemSpaX can place substituents on specific sites of diverse molecular scaffolds based on initial user input and uses FF optimization to optimize newly placed substituents. Use cases were shown by using a data-augmented approach which utilized fast GFN2-xTB optimizations to compare structures generated by ChemSpaX. For selected use cases a comparison was also done against DFT optimized structures. Descriptors such as the (free) energy of reaction that can be used for HTS applications were studied in more detail for some of the presented use cases. For the pincer complexes the quality of geometries generated by ChemSpaX was assessed along the energy $(\Delta \Delta \mathrm{E})$ and spatial (hRMSDs) dimensions. Generally FF geometries were found to be similar in quality compared to GFN2-xTB optimized geometries based on the hRMSDs, and $\Delta \Delta$ E. Intramolecular movements pose a challenge for FF geometries which show high hRMSDs for complexes involving labile ligands. A use case of ChemSpaX enabled QM calculations was 
the discovery of a strong linear scaling of $\Delta \mathrm{E}$ and $\Delta \mathrm{G}\left(\mathrm{R}^{2}=0.99\right.$ for both RuPNP and the Mnpincers). This correlation was found for both DFT and GFN2-xTB calculations allowing lower computational costs by skipping the calculation of hessian matrices to estimate $\Delta \mathrm{G}$. Analysis of functionalized Co porphyrins generated by ChemSpaX showed that the hRMSD increased linearly with the number of atoms in serial functionalization runs on a given skeleton. This observation paves the way for devising a strategy to optimally employ higher-level geometry optimizations in intermediate steps. hRMSD was found to be a potential feature in advanced machine learning applications to predict the HOMO-LUMO gap. It has been demonstrated that diverse molecular scaffolds can be functionalized using ChemSpaX.

To conclude, ChemSpaX can be used to generate satisfactory 3D geometric representations in the local chemical space of a given molecular scaffold, particularly including TM complexes. The generated structures, in conjunction with quantum chemical and statistical methods, can be used to generate structure-property databases enabling data-driven chemical design and discovery. We are working to further improve ChemSpaX along diverse research lines. Improved force-field methods can definitely help improve the accuracy of ChemSpaX. A data-informed approach to estimate the hRMSD based on the identity of the skeleton molecule and functional groups placed can improve the predictive capabilities for screening applications. Parallelization of the code for placing functional groups is work in progress. Additionally, we are working on implementing a more flexible approach where the user can choose the frequency of intermediate optimizations with a high-level method based on a predicted hRMSD threshold, and also get recommendations about optimal strategies balancing speed and accuracy.

With the development of ChemSpaX, an important challenge in HTS related to the generation of reasonably accurate 3D molecular geometries is addressed. This workflow is aimed at accelerating chemical space exploration and we hope that ChemSpaX will bolster and democratize the efforts of the catalysis and molecular modelling communities towards data-driven material discovery. ChemSpaX is free, open-source and is available with an introductory Google colaboratory notebook which can be immediately used by researchers.

\section{Data and software availability}

The ChemSpaX workflow will be made public on our Github organization page: EPiCs-group (https://github.com/EPiCs-group). In addition to this manuscript, supporting information and all used datasets can be found:

- full_datasets.zip contains datasets per investigated complex in Excel workbook format.

- geometry_files.zip contains geometry files for all structures in MDL Molfiles or $X Y Z$ format.

\section{Author contributions}

The code for ChemSpaX was written by A. V. K. and V. S. DFT \& XTB calculations were performed by A. V. K and V. S. Generation of functionalized structures, the compilation of datasets and analysis of DFT \& XTB calculations was performed by A. V. K. under supervision of V. S. V. S. and E. A. P. conceived the project. E. A. P. played an advisory role and directed the project. All the authors discussed the results and wrote the manuscript.

\section{Conflicts of interest}

There are no conflicts of interest to declare. 


\section{Acknowledgments}

V. S. acknowledges the ARC-CBBC project 2016.008 for funding. E.A.P. acknowledges the financial support from the European Research Council (ERC) under the European Union's Horizon 2020 Research and Innovation Programme (grant agreement no. 725686). This work was sponsored by NWO Domain Science for the use of the national computer facilities. We acknowledge that the results of this research have been partially achieved using the $\mathrm{DECl}$ resource, Kay, based in Ireland at ICHEC with support from PRACE under the DECI16 call. The authors thank the PetaChem team for giving access to the TeraChem software package.

\section{References}

[1] John H Van Drie. Computer-aided drug design: the next 20 years. Journal of ComputerAided Molecular Design, 21(10):591-601, 2007. ISSN 1573-4951. doi: 10.1007/s10822007-9142-y. URL https://doi.org/10.1007/s10822-007-9142-y.

[2] Gregory Sliwoski, Sandeepkumar Kothiwale, Jens Meiler, and Edward W Lowe Jr. Computational methods in drug discovery. Pharmacological reviews, 66(1):334-395, dec 2013. ISSN 1521-0081. doi: 10.1124/pr.112.007336. URL https://pubmed.ncbi.nlm.nih. gov/24381236https://www.ncbi.nlm.nih.gov/pmc/articles/PMC3880464/.

[3] David E Clark. What has computer-aided molecular design ever done for drug discovery? Expert Opinion on Drug Discovery, 1(2):103-110, jul 2006. ISSN 1746-0441. doi: 10. 1517/17460441.1.2.103. URL https://doi.org/10.1517/17460441.1.2.103.

[4] David E Clark. What has virtual screening ever done for drug discovery? Expert Opinion on Drug Discovery, 3(8):841-851, aug 2008. ISSN 1746-0441. doi: 10.1517/17460441.3. 8.841. URL https://doi.org/10.1517/17460441.3.8.841.

[5] Anubhav Jain, Yongwoo Shin, and Kristin A Persson. Computational predictions of energy materials using density functional theory. Nature Reviews Materials, 1(1):15004, 2016. ISSN 2058-8437. doi: 10.1038/natrevmats.2015.4. URL https://doi.org/10.1038/ natrevmats.2015.4.

[6] Lei Cheng, Rajeev S Assary, Xiaohui Qu, Anubhav Jain, Shyue Ping Ong, Nav Nidhi Rajput, Kristin Persson, and Larry A Curtiss. Accelerating Electrolyte Discovery for Energy Storage with High-Throughput Screening. The Journal of Physical Chemistry Letters, 6 (2):283-291, jan 2015. doi: 10.1021/jz502319n. URL https://doi.org/10.1021/ jz502319n.

[7] Hossein Beidaghy Dizaji and Hannaneh Hosseini. A review of material screening in pure and mixed-metal oxide thermochemical energy storage (TCES) systems for concentrated solar power (CSP) applications. Renewable and Sustainable Energy Reviews, 98:9-26, 2018. ISSN 1364-0321. doi: https://doi.org/10.1016/j.rser.2018.09.004. URL http: //www.sciencedirect.com/science/article/pii/S136403211830652X.

[8] Johannes Hachmann, Roberto Olivares-Amaya, Sule Atahan-Evrenk, Carlos AmadorBedolla, Roel S Sánchez-Carrera, Aryeh Gold-Parker, Leslie Vogt, Anna M Brockway, and Alán Aspuru-Guzik. The Harvard Clean Energy Project: Large-Scale Computational Screening and Design of Organic Photovoltaics on the World Community Grid. The Journal of Physical Chemistry Letters, 2(17):2241-2251, sep 2011. doi: 10.1021/jz200866s. URL https://doi.org/10.1021/jz200866s. 
[9] Natalie Fey. Lost in chemical space? maps to support organometallic catalysis. Chemistry Central Journal, 9(1):38, Jun 2015. ISSN 1752-153X. doi: 10.1186/s13065-015-0104-5. URL https://doi.org/10.1186/s13065-015-0104-5.

[10] Peter Kirkpatrick and Clare Ellis. Chemical space. Nature, 432(7019):823, 2004. ISSN 1476-4687. doi: 10.1038/432823a. URL https://doi.org/10.1038/432823a.

[11] Fernanda I Saldívar-González, B Angélica Pilón-Jiménez, and José L Medina-Franco. Chemical space of naturally occurring compounds. Physical Sciences Reviews, 4(5), 2019. doi: doi:10.1515/psr-2018-0103. URL https://doi.org/10.1515/psr-2018-0103.

[12] Christopher Zhou, William Grumbles, and Thomas Cundari. Using Machine Learning to Predict the pKa of $\mathrm{C}-\mathrm{H}$ Bonds. Relevance to Catalytic Methane Functionalization, jul 2020. URL https://chemrxiv.org/articles/preprint/Using_Machine_ Learning_to_Predict_the_pKa_of_C_H_Bonds_Relevance_to_Catalytic_ Methane_Functionalization/12646772https://chemrxiv.org/ndownloader/ files/23820425.

[13] O Anatole von Lilienfeld, Klaus-Robert Müller, and Alexandre Tkatchenko. Exploring chemical compound space with quantum-based machine learning. Nature Reviews Chemistry, 4(7):347-358, 2020. ISSN 2397-3358. doi: 10.1038/s41570-020-0189-9. URL https://doi.org/10.1038/s41570-020-0189-9.

[14] Tobias Fink and Jean-Louis Reymond. Virtual Exploration of the Chemical Universe up to 11 Atoms of C, N, O, F: Assembly of 26.4 Million Structures (110.9 Million Stereoisomers) and Analysis for New Ring Systems, Stereochemistry, Physicochemical Properties, Compound Classes, and Drug Discovery. Journal of Chemical Information and Modeling, 47(2):342-353, mar 2007. ISSN 1549-9596. doi: 10.1021/ci600423u. URL https://doi.org/10.1021/ci600423u.

[15] Lorenz C Blum and Jean-Louis Reymond. 970 Million Druglike Small Molecules for Virtual Screening in the Chemical Universe Database GDB-13. Journal of the American Chemical Society, 131(25):8732-8733, jul 2009. ISSN 0002-7863. doi: 10.1021/ja902302h. URL https://doi.org/10.1021/ja902302h.

[16] Lars Ruddigkeit, Ruud van Deursen, Lorenz C Blum, and Jean-Louis Reymond. Enumeration of 166 Billion Organic Small Molecules in the Chemical Universe Database GDB-17. Journal of Chemical Information and Modeling, 52(11):2864-2875, nov 2012. ISSN 15499596. doi: 10.1021/ci300415d. URL https://doi.org/10.1021/ci300415d.

[17] Teague Sterling and John J Irwin. ZINC 15 - Ligand Discovery for Everyone. Journal of Chemical Information and Modeling, 55(11):2324-2337, nov 2015. ISSN 1549-9596. doi: 10.1021/acs.jcim.5b00559. URL https://doi.org/10.1021/acs.jcim.5b00559.

[18] Josep Arús-Pous, Thomas Blaschke, Silas Ulander, Jean-Louis Reymond, Hongming Chen, and Ola Engkvist. Exploring the GDB-13 chemical space using deep generative models. Journal of Cheminformatics, 11(1):20, 2019. ISSN 1758-2946. doi: 10.1186/s13321-0190341-z. URL https://doi.org/10.1186/s13321-019-0341-z.

[19] Oliver T. Unke, Stefan Chmiela, Huziel E. Sauceda, Michael Gastegger, Igor Poltavsky, Kristof T. Schütt, Alexandre Tkatchenko, and Klaus-Robert Müller. Machine learning force fields. Chemical Reviews, Mar 2021. ISSN 0009-2665. doi: 10.1021/acs.chemrev.0c01111. URL https://doi.org/10.1021/acs . chemrev.0c01111. 
[20] Fabio Begnini, Vasanthanathan Poongavanam, Björn Over, Marie Castaldo, Stefan Geschwindner, Patrik Johansson, Mohit Tyagi, Christian Tyrchan, Lisa Wissler, Peter Sjö, Stefan Schiesser, and Jan Kihlberg. Mining natural products for macrocycles to drug difficult targets. Journal of Medicinal Chemistry, 64:1054-1072, 12 2020. doi: 10.1021/acs.jmedchem.0c01569.

[21] Adrian Jinich, Benjamin Sanchez-Lengeling, Haniu Ren, Rebecca Harman, and Alán Aspuru-Guzik. A Mixed Quantum Chemistry/Machine Learning Approach for the Fast and Accurate Prediction of Biochemical Redox Potentials and Its Large-Scale Application to 315000 Redox Reactions. ACS Central Science, 5(7):1199-1210, jul 2019. ISSN 2374-7943. doi: 10.1021/acscentsci.9b00297. URL https://doi.org/10.1021/ acscentsci.9b00297.

[22] Rodney A. Fernandes, Amit K. Jha, and Praveen Kumar. Recent advances in wacker oxidation: From conventional to modern variants and applications. Catalysis Science and Technology, 10:7448-7470, 11 2020. ISSN 20444761. doi: 10.1039/d0cy01820a. URL http://ether.chem.iitb.ac.in/ rfernand/.

[23] Matthew L. Crawley and Barry M. Trost. Applications of Transition Metal Catalysis in Drug Discovery and Development: An Industrial Perspective. John Wiley and Sons, 5 2012. ISBN 9780470631324. doi: 10.1002/9781118309872. URL http://www.wiley. com/go/permission. www . wiley.com.

[24] Wilhelm Keim. Concepts for the Use of Transition Metals in Industrial Fine Chemical Synthesis. Wiley-VCH Verlag GmbH, 2 2008. doi: 10.1002/9783527619405.ch1b. URL https://onlinelibrary.wiley.com/doi/abs/10.1002/9783527619405.ch1b.

[25] Wataru Kuriyama, Takaji Matsumoto, Osamu Ogata, Yasunori Ino, Kunimori Aoki, Shigeru Tanaka, Kenya Ishida, Tohru Kobayashi, Noboru Sayo, and Takao Saito. Catalytic hydrogenation of esters. development of an efficient catalyst and processes for synthesising (r)-1,2-propanediol and 2-(I-menthoxy)ethanol. Organic Process Research and Development, 16:166-171, 1 2012. ISSN 10836160. doi: 10.1021/op200234j. URL https://pubs . acs .org/sharingguidelines.

[26] Ba L. Tran, Samantha I. Johnson, Kriston P. Brooks, and S. Tom Autrey. Ethanol as a liquid organic hydrogen carrier for seasonal microgrid application: Catalysis, theory, and engineering feasibility. ACS Sustainable Chemistry \& Engineering, page acssuschemeng.1c01513, 5 2021. ISSN 2168-0485. doi: 10.1021/acssuschemeng.1c01513. URL https://pubs.acs.org/doi/10.1021/acssuschemeng.1c01513.

[27] Jens Hagen. Industrial Catalysis: A Practical Approach, chapter Homogeneously Catalyzed Industrial Processes, pages 47-80. Wiley-VCH Verlag GmbH \& Co. KGaA, 9 2015. doi: 10.1002/9783527684625.ch3.

[28] Gabriel dos Passos Gomes, Robert Pollice, and Alán Aspuru-Guzik. Navigating through the Maze of Homogeneous Catalyst Design with Machine Learning. Trends in Chemistry, 3(2):96-110, 2021. ISSN 2589-5974. doi: https://doi.org/10.1016/ j.trechm.2020.12.006. URL https://www.sciencedirect.com/science/article/ pii/S2589597420303166.

[29] Robert Franke, Detlef Selent, and Armin Börner. Applied hydroformylation. Chemical 
Reviews, 112:5675-5732, 11 2012. ISSN 00092665. doi: 10.1021/cr3001803. URL https://pubs .acs.org/sharingguidelines.

[30] Stefan Gugler, Jon Paul Janet, and Heather J. Kulik. Enumeration of de novo inorganic complexes for chemical discovery and machine learning. Mol. Syst. Des. Eng., 5:139-152, 2020. doi: 10.1039/C9ME00069K. URL http://dx.doi.org/10.1039/C9ME00069K.

[31] Jeremy J. Henle, Andrew F. Zahrt, Brennan T. Rose, William T. Darrow, Yang Wang, and Scott E. Denmark. Development of a computer-guided workflow for catalyst optimization. descriptor validation, subset selection, and training set analysis. Journal of the American Chemical Society, 142:11578-11592, 7 2020. ISSN 15205126. doi: 10.1021/jacs.0c04715. URL https://pubs.acs.org/doi/full/10.1021/jacs.0c04715.

[32] Jan Grimo Sobez and Markus Reiher. Molassembler: Molecular graph construction, modification, and conformer generation for inorganic and organic molecules. Journal of Chemical Information and Modeling, 60:3884-3900, 8 2020. ISSN 15205142. doi: 10.1021/acs. jcim.0c00503. URL https://pubs.acs.org/doi/full/10.1021/acs.jcim.0c00503.

[33] Derek J. Durand and Natalie Fey. Building a toolbox for the analysis and prediction of ligand and catalyst effects in organometallic catalysis. Accounts of Chemical Research, 54:837-848, 2 2021. ISSN 15204898. doi: 10.1021/acs.accounts.0c00807. URL https: //pubs.acs.org/doi/full/10.1021/acs.accounts.0c00807.

[34] David Balcells and Bastian Bjerkem Skjelstad. Tmqm dataset - quantum geometries and properties of $86 \mathrm{k}$ transition metal complexes. Journal of Chemical Information and Modeling, 60:6135-6146, 12 2020. ISSN 15205142. doi: 10.1021/acs.jcim.0c01041. URL https://dx.doi.org/10.1021/acs.jcim.0c01041.

[35] Felix J. De Zwart, Bente Reus, Annechien A.H. Laporte, Vivek Sinha, and Bas De Bruin. Metrical oxidation states of 1,4-diazadiene-derived ligands. Inorganic Chemistry, 60:32743281, 3 2021. ISSN 1520510X. doi: 10.1021/acs.inorgchem.0c03685. URL https: //dx.doi.org/10.1021/acs.inorgchem.0c03685.

[36] Adam I. Green, Christopher P. Tinworth, Stuart Warriner, Adam Nelson, and Natalie Fey. Computational mapping of dirhodium(ii) catalysts. Chemistry - A European Journal, 27(7):2402-2409, 2021. doi: https://doi.org/10.1002/chem. 202003801. URL https://chemistry-europe.onlinelibrary.wiley.com/doi/abs/ 10.1002/chem. 202003801.

[37] Pascal Friederich, Gabriel dos Passos Gomes, Riccardo De Bin, Alán Aspuru-Guzik, and David Balcells. Machine learning dihydrogen activation in the chemical space surrounding vaska's complex. Chem. Sci., 11:4584-4601, 2020. doi: 10.1039/D0SC00445F. URL http://dx.doi.org/10.1039/D0SC00445F.

[38] Kaid C. Harper, Elizabeth N. Bess, and Matthew S. Sigman. Multidimensional steric parameters in the analysis of asymmetric catalytic reactions. Nature Chemistry, 4:366374, 5 2012. ISSN 17554330. doi: 10.1038/nchem.1297. URL ww.nature.com/ naturechemistry.

[39] Jolene P. Reid, Rupert S.J. Proctor, Matthew S. Sigman, and Robert J. Phipps. Predictive multivariate linear regression analysis guides successful catalytic enantioselective minisci reactions of diazines. Journal of the American Chemical Society, 141:19178-19185, 12 
2019. ISSN 15205126. doi: 10.1021/jacs.9b11658. URL https://pubs.acs.org/ sharingguidelines.

[40] Celine B. Santiago, Jing Yao Guo, and Matthew S. Sigman. Predictive and mechanistic multivariate linear regression models for reaction development. Chemical Science, 9:2398-2412, 3 2018. ISSN 20416539. doi: 10.1039/c7sc04679k. URL https://pubs.rsc.org/en/content/articlehtml/2018/sc/c7sc04679khttps:

//pubs.rsc.org/en/content/articlelanding/2018/sc/c7sc04679k.

[41] Jolene P. Reid and Matthew S. Sigman. Holistic prediction of enantioselectivity in asymmetric catalysis. Nature, 571:343-348, 7 2019. ISSN 14764687. doi: 10.1038/s41586019-1384-z. URL https://doi.org/10.1038/s41586-019-1384-z.

[42] David Weininger. Smiles, a chemical language and information system: 1: Introduction to methodology and encoding rules. Journal of Chemical Information and Computer Sciences, 28:31-36, 2 1988. ISSN 00952338. doi: 10.1021/ci00057a005. URL https: //dl.acm.org/doi/abs/10.1021/ci00057a005.

[43] Craig A James, Richard Apodaca, Noel M O'Boyle, Andrew Dalke, John H Van Drie, Peter Ertl, Geoffrey R Hutchison, Greg Landrum, Chris Morley, Egon Willighagen, Hans De winter, Tim Vandermeersch, and John May. OpenSMILES specification, 2016. URL http://opensmiles.org/opensmiles.html.

[44] Jan Jensen. xyz2mol: Convert Cartesian coordinates to one or more molecular graphs, 2020. URL https://github.com/jensengroup/xyz2mol.

[45] Mario Krenn, Florian Häse, AkshatKumar Nigam, Pascal Friederich, and Alan AspuruGuzik. Self-referencing embedded strings (SELFIES): A 100\% robust molecular string representation. Machine Learning: Science and Technology, 1(4):45024, 2020. ISSN 26322153. doi: 10.1088/2632-2153/aba947. URL http://dx.doi.org/10.1088/2632$2153 /$ aba947.

[46] Efthymios I loannidis, Terry Z H Gani, and Heather J Kulik. molSimplify: A toolkit for automating discovery in inorganic chemistry. Journal of Computational Chemistry, 37(22): 2106-2117, 2016. ISSN 1096-987X. doi: 10.1002/jcc.24437. URL http://dx.doi.org/ $10.1002 / \mathrm{jcc} .24437$.

[47] Jon Paul Janet, Fang Liu, Aditya Nandy, Chenru Duan, Tzuhsiung Yang, Sean Lin, and Heather J Kulik. Designing in the Face of Uncertainty: Exploiting Electronic Structure and Machine Learning Models for Discovery in Inorganic Chemistry. Inorganic Chemistry, 58(16):10592-10606, aug 2019. ISSN 0020-1669. doi: 10.1021/acs.inorgchem.9b00109. URL https://doi.org/10.1021/acs.inorgchem.9b00109.

[48] Aditya Nandy, Chenru Duan, Jon Paul Janet, Stefan Gugler, and Heather J Kulik. Strategies and Software for Machine Learning Accelerated Discovery in Transition Metal Chemistry. Industrial \& Engineering Chemistry Research, 57(42):13973-13986, oct 2018. ISSN 0888-5885. doi: 10.1021/acs.iecr.8b04015. URL https://doi.org/10.1021/acs. iecr. 8b04015.

[49] Jon Paul Janet, Terry Z H Gani, Adam H Steeves, Efthymios I loannidis, and Heather J Kulik. Leveraging Cheminformatics Strategies for Inorganic Discovery: Application to Redox Potential Design. Industrial \& Engineering Chemistry Research, 56(17):4898-4910, 
may 2017. ISSN 0888-5885. doi: 10.1021/acs.iecr.7b00808. URL https://doi.org/ 10.1021/acs.iecr.7b00808.

[50] Jon Paul Janet, Qing Zhao, Efthymios I loannidis, and Heather J Kulik. Density functional theory for modelling large molecular adsorbate-surface interactions: a mini-review and worked example. Molecular Simulation, 43(5-6):327-345, apr 2017. ISSN 0892-7022. doi: 10.1080/08927022.2016.1258465. URL https://doi.org/10.1080/08927022.2016. 1258465.

[51] Victoria M. Ingman, Anthony J. Schaefer, Laura R. Andreola, and Steven E. Wheeler. Qchasm: Quantum chemistry automation and structure manipulation. Wiley Interdisciplinary Reviews: Computational Molecular Science, 2020. ISSN 17590884. doi: $10.1002 /$ wcms. 1510.

[52] Lukas Turcani, Andrew Tarzia, Filip T. Szczypiński, and Kim E. Jelfs. stk: An extendable python framework for automated molecular and supramolecular structure assembly and discovery. The Journal of Chemical Physics, 154(21):214102, 2021. doi: 10.1063/5. 0049708. URL https://doi.org/10.1063/5.0049708.

[53] Luca Piccirilli, Danielle Lobo Justo Pinheiro, and Martin Nielsen. Recent progress with pincer transition metal catalysts for sustainability. Catalysts, 10:773, 72020 . ISSN 20734344. doi: 10.3390/catal10070773. URL www.mdpi.com/journal/catalysts.

[54] Gerard Parkin. Special issue on pincer ligands. Polyhedron, 143:1, 3 2018. ISSN 02775387. doi: $10.1016 /$ j.poly.2018.02.019.

[55] Leon Maser, Lisa Vondung, and Robert Langer. The abc in pincer chemistry - from amine- to borylene- and carbon-based pincer-ligands. Polyhedron, 143:28-42, 32018. ISSN 02775387. doi: 10.1016/j.poly.2017.09.009.

[56] Mark A.W. Lawrence, Kerry Ann Green, Peter N. Nelson, and Shannen C. Lorraine. Review: Pincer ligands-tunable, versatile and applicable. Polyhedron, 143:11-27, 32018. ISSN 02775387. doi: 10.1016/j.poly.2017.08.017.

[57] Sudakar Padmanaban, Gunniya Hariyanandam Gunasekar, and Sungho Yoon. Direct heterogenization of the ru-macho catalyst for the chemoselective hydrogenation of , unsaturated carbonyl compounds. Inorganic Chemistry, 60:6881-6888, 2021. ISSN 1520510X. doi: 10.1021/acs.inorgchem.0c03681. URL https://dx.doi.org/10.1021/ acs. inorgchem.0c03681.

[58] Pavel A. Dub and John C. Gordon. The role of the metal-bound $\mathrm{n}-\mathrm{h}$ functionality in noyori-type molecular catalysts. Nature Reviews Chemistry, 2:396-408, 12 2018. ISSN 23973358. doi: 10.1038/s41570-018-0049-z. URL www.nature.com/natrevchem.

[59] Georgy A. Filonenko, Robbert Van Putten, Emiel J.M. Hensen, and Evgeny A. Pidko. Catalytic (de)hydrogenation promoted by non-precious metals-co, fe and $\mathrm{mn}$ : Recent advances in an emerging field. Chemical Society Reviews, 47:1459-1483, 2 2018. ISSN 14604744. doi: 10.1039/c7cs00334j. URL https://pubs.rsc.org/en/content/articlehtml/2018/cs/c7cs00334jhttps: //pubs.rsc.org/en/content/articlelanding/2018/cs/c7cs00334j.

[60] Jose R. Cabrero-Antonino, Rosa Adam, Veronica Papa, and Matthias Beller. Homogeneous and heterogeneous catalytic reduction of amides and related compounds using molecular 
hydrogen. Nature Communications, 11:1-18, 12 2020. ISSN 20411723. doi: 10.1038/ s41467-020-17588-5. URL https://doi.org/10.1038/s41467-020-17588-5.

[61] James Pritchard, Georgy A. Filonenko, Robbert Van Putten, Emiel J.M. Hensen, and Evgeny A. Pidko. Heterogeneous and homogeneous catalysis for the hydrogenation of carboxylic acid derivatives: History, advances and future directions. Chemical Society Reviews, 44:3808-3833, 6 2015. ISSN 14604744. doi: 10.1039/c5cs00038f. URL www. rsc.org/csr.

[62] Robbert van Putten, Tim Wissink, Tijn Swinkels, and Evgeny A. Pidko. Fuelling the hydrogen economy: Scale-up of an integrated formic acid-to-power system. International Journal of Hydrogen Energy, 44:28533-28541, 11 2019. ISSN 03603199. doi: 10.1016/j. ijhydene.2019.01.153.

[63] Annika Krieger, Vivek Sinha, Adarsh Kalikadien, and Evgeny Alexandrovich Pidko. Metalligand cooperative activation of $\mathrm{HX}(\mathrm{X}=\mathrm{H}, \mathrm{Br}, \mathrm{OR})$ bond on $\mathrm{Mn}$ based pincer complexes. Zeitschrift für anorganische und allgemeine Chemie, $\mathrm{n} / \mathrm{a}(\mathrm{n} / \mathrm{a})$, may 2021. ISSN 0044-2313. doi: https://doi.org/10.1002/zaac.202100078. URL https://doi.org/10. 1002/zaac. 202100078.

[64] Marcel Garbe, Kathrin Junge, and Matthias Beller. Homogeneous Catalysis by ManganeseBased Pincer Complexes. European Journal of Organic Chemistry, 2017(30):4344-4362, aug 2017. ISSN 1434-193X. doi: https://doi.org/10.1002/ejoc.201700376. URL https: //doi.org/10.1002/ejoc. 201700376.

[65] Wenjun Yang, Ivan Yu Chernyshov, Robin K.A. van Schendel, Manuela Weber, Christian Müller, Georgy A. Filonenko, and Evgeny A. Pidko. Robust and efficient hydrogenation of carbonyl compounds catalysed by mixed donor mn(i) pincer complexes. Nature Communications, 12:1-8, 12 2021. ISSN 20411723. doi: 10.1038/s41467-020-20168-2. URL https://doi.org/10.1038/s41467-020-20168-2.

[66] Lukas Alig, Maximilian Fritz, and Sven Schneider. First-row transition metal (de)hydrogenation catalysis based on functional pincer ligands. Chemical Reviews, 119:2681-2751, 2 2019. ISSN 15206890. doi: 10.1021/acs.chemrev.8b00555. URL https://pubs.acs.org/sharingguidelines.

[67] Monalisa Goswami, Christophe Rebreyend, and Bas de Bruin. Porphyrin Cobalt(III) "Nitrene Radical" Reactivity; Hydrogen Atom Transfer from Ortho-YH Substituents to the Nitrene Moiety of Cobalt-Bound Aryl Nitrene Intermediates $(\mathrm{Y}=\mathrm{O}, \mathrm{NH})$. Molecules, 21(2):242, feb 2016. ISSN 1420-3049. doi: 10.3390/molecules21020242. URL http: //dx.doi.org/10.3390/molecules21020242.

[68] Michael P Doyle and David C Forbes. Recent Advances in Asymmetric Catalytic Metal Carbene Transformations. Chemical Reviews, 98(2):911-936, apr 1998. ISSN 0009-2665. doi: 10.1021/cr940066a. URL https://doi.org/10.1021/cr940066a.

[69] Simone Fantauzzi, Alessandro Caselli, and Emma Gallo. Nitrene transfer reactions mediated by metallo-porphyrin complexes. Dalton Transactions, (28):5434-5443, 2009. ISSN 1477-9226. doi: 10.1039/B902929J. URL http://dx.doi.org/10.1039/B902929J.

[70] Matthias Otte, Petrus F Kuijpers, Oliver Troeppner, Ivana Ivanović-Burmazović, Joost N H Reek, and Bas de Bruin. Encapsulated Cobalt-Porphyrin as a Catalyst for Size-Selective 
Radical-type Cyclopropanation Reactions. Chemistry - A European Journal, 20(17):48804884, apr 2014. ISSN 0947-6539. doi: https://doi.org/10.1002/chem.201400055. URL https://doi.org/10.1002/chem. 201400055.

[71] Valentinos Mouarrawis, Raoul Plessius, Jarl Ivar van der Vlugt, and Joost N H Reek. Confinement Effects in Catalysis Using Well-Defined Materials and Cages, 2018. URL https://www.frontiersin.org/article/10.3389/fchem.2018.00623.

[72] Matthias Otte, Petrus F Kuijpers, Oliver Troeppner, Ivana Ivanović-Burmazović, Joost N H Reek, and Bas de Bruin. Encapsulation of Metalloporphyrins in a Self-Assembled Cubic M8L6 Cage: A New Molecular Flask for Cobalt-Porphyrin-Catalysed Radical-Type Reactions. Chemistry - A European Journal, 19(31):10170-10178, jul 2013. ISSN 09476539. doi: https://doi.org/10.1002/chem.201301411. URL https://doi.org/10. 1002/chem. 201301411.

[73] Noel M O'Boyle, Michael Banck, Craig A James, Chris Morley, Tim Vandermeersch, and Geoffrey R Hutchison. The Open Babel Package, version 2.4.1, 2016. URL https: //openbabel.org/.

[74] Noel M O'Boyle, Michael Banck, Craig A James, Chris Morley, Tim Vandermeersch, and Geoffrey R Hutchison. Open Babel: An open chemical toolbox. Journal of Cheminformatics, 3(1):33, 2011. ISSN 1758-2946. doi: 10.1186/1758-2946-3-33. URL https://doi.org/10.1186/1758-2946-3-33.

[75] A K Rappe, C J Casewit, K S Colwell, W A Goddard, and W M Skiff. UFF, a full periodic table force field for molecular mechanics and molecular dynamics simulations. Journal of the American Chemical Society, 114(25):10024-10035, dec 1992. ISSN 0002-7863. doi: 10.1021/ja00051a040. URL https://doi.org/10.1021/ja00051a040.

[76] Junmei Wang, Romain M Wolf, James W Caldwell, Peter A Kollman, and David A Case. Development and testing of a general amber force field. Journal of Computational Chemistry, 25(9):1157-1174, jul 2004. ISSN 0192-8651. doi: https://doi.org/10.1002/jcc. 20035. URL https://doi.org/10.1002/jcc. 20035.

[77] Christoph Bannwarth, Eike Caldeweyher, Sebastian Ehlert, Andreas Hansen, Philipp Pracht, Jakob Seibert, Sebastian Spicher, and Stefan Grimme. Extended tight-binding quantum chemistry methods. WIREs Computational Molecular Science, n/a(n/a):e01493, aug 2020. ISSN 1759-0876. doi: 10.1002/wcms.1493. URL https://doi.org/10.1002/ wcms . 1493.

[78] Philipp Pracht, Eike Caldeweyher, Sebastian Ehlert, and Stefan Grimme. A Robust Non-Self-Consistent Tight-Binding Quantum Chemistry Method for large Molecules. ChemRxiv, jun 2019. doi: 10.26434/chemrxiv.8326202.v1. URL https: //chemrxiv.org/articles/preprint/A_Robust_Non-Self-Consistent_TightBinding_Quantum_Chemistry_Method_for_large_Molecules/8326202https: //chemrxiv.org/ndownloader/files/15605534.

[79] Stefan Grimme, Christoph Bannwarth, and Philip Shushkov. A Robust and Accurate Tight-Binding Quantum Chemical Method for Structures, Vibrational Frequencies, and Noncovalent Interactions of Large Molecular Systems Parametrized for All spd-Block Elements $(Z=1-86)$. Journal of Chemical Theory and Computation, 13(5):1989- 
2009, may 2017. ISSN 1549-9618. doi: 10.1021/acs.jctc.7b00118. URL https: //doi.org/10.1021/acs.jctc.7b00118.

[80] Christoph Bannwarth, Sebastian Ehlert, and Stefan Grimme. GFN2-xTB-An Accurate and Broadly Parametrized Self-Consistent Tight-Binding Quantum Chemical Method with Multipole Electrostatics and Density-Dependent Dispersion Contributions. Journal of Chemical Theory and Computation, 15(3):1652-1671, mar 2019. ISSN 1549-9618. doi: 10.1021/acs.jctc.8b01176. URL https://doi.org/10.1021/acs.jctc.8b01176.

[81] Sebastian Spicher and Stefan Grimme. Robust Atomistic Modeling of Materials, Organometallic, and Biochemical Systems. Angewandte Chemie International Edition, 59(36):15665-15673, sep 2020. ISSN 1433-7851. doi: https://doi.org/10.1002/anie. 202004239. URL https://doi.org/10.1002/anie.202004239.

[82] W Clark Still, Anna Tempczyk, Ronald C Hawley, and Thomas Hendrickson. Semianalytical treatment of solvation for molecular mechanics and dynamics. Journal of the American Chemical Society, 112(16):6127-6129, aug 1990. ISSN 0002-7863. doi: 10.1021/ja00172a038. URL https://doi.org/10.1021/ja00172a038.

[83] T Ooi, M Oobatake, G Némethy, and H A Scheraga. Accessible surface areas as a measure of the thermodynamic parameters of hydration of peptides. Proceedings of the National Academy of Sciences, 84(10):3086 LP - 3090, may 1987. doi: 10.1073/pnas.84.10.3086. URL http://www . pnas.org/content/84/10/3086. abstract.

[84] M J Frisch, G W Trucks, H B Schlegel, G E Scuseria, M A Robb, J R Cheeseman, G Scalmani, V Barone, G A Petersson, H Nakatsuji, X Li, M Caricato, A V Marenich, J Bloino, B G Janesko, R Gomperts, B Mennucci, H P Hratchian, J V Ortiz, A F Izmaylov, J L Sonnenberg, D Williams-Young, F Ding, F Lipparini, F Egidi, J Goings, B Peng, A Petrone, T Henderson, D Ranasinghe, V G Zakrzewski, J Gao, N Rega, G Zheng, W Liang, M Hada, M Ehara, K Toyota, R Fukuda, J Hasegawa, M Ishida, T Nakajima, Y Honda, O Kitao, H Nakai, T Vreven, K Throssell, J A Montgomery Jr., J E Peralta, F Ogliaro, M J Bearpark, J J Heyd, E N Brothers, K N Kudin, V N Staroverov, T A Keith, R Kobayashi, J Normand, K Raghavachari, A P Rendell, J C Burant, S S lyengar, J Tomasi, M Cossi, J M Millam, M Klene, C Adamo, R Cammi, J W Ochterski, R L Martin, K Morokuma, O Farkas, J B Foresman, and D J Fox. Gaussian 16 Revision C.01, 2016.

[85] A D Becke. Density-functional exchange-energy approximation with correct asymptotic behavior. Physical Review A, 38(6):3098-3100, sep 1988. doi: 10.1103/PhysRevA.38. 3098. URL https://link.aps.org/doi/10.1103/PhysRevA.38.3098.

[86] Florian Weigend and Reinhart Ahlrichs. Balanced basis sets of split valence, triple zeta valence and quadruple zeta valence quality for $\mathrm{H}$ to $\mathrm{Rn}$ : Design and assessment of accuracy. Physical Chemistry Chemical Physics, 7(18):3297-3305, 2005. ISSN 1463-9076. doi: 10.1039/B508541A. URL http://dx.doi.org/10.1039/B508541A.

[87] Kasper P Jensen, Björn O Roos, and Ulf Ryde. Performance of density functionals for first row transition metal systems. The Journal of Chemical Physics, 126(1):14103, jan 2007. ISSN 0021-9606. doi: 10.1063/1.2406071. URL https://doi.org/10.1063/1. 2406071.

[88] Michael Bühl and Hendrik Kabrede. Geometries of Transition-Metal Complexes from 
Density-Functional Theory. Journal of Chemical Theory and Computation, 2(5):12821290, sep 2006. ISSN 1549-9618. doi: 10.1021/ct6001187. URL https://doi.org/10. $1021 /$ ct6001187.

[89] Aleksandr V Marenich, Christopher J Cramer, and Donald G Truhlar. Universal Solvation Model Based on Solute Electron Density and on a Continuum Model of the Solvent Defined by the Bulk Dielectric Constant and Atomic Surface Tensions. The Journal of Physical Chemistry B, 113(18):6378-6396, may 2009. ISSN 1520-6106. doi: 10.1021/jp810292n. URL https://doi.org/10.1021/jp810292n.

[90] Carlo Adamo and Vincenzo Barone. Toward reliable density functional methods without adjustable parameters: The pbe0 model. Journal of Chemical Physics, 110:6158-6170, 4 1999. ISSN 00219606. doi: 10.1063/1.478522. URL https://doi.org/10.1063/1. 478522.

[91] Eike Caldeweyher, Sebastian Ehlert, Andreas Hansen, Hagen Neugebauer, Sebastian Spicher, Christoph Bannwarth, and Stefan Grimme. A generally applicable atomiccharge dependent London dispersion correction. The Journal of Chemical Physics, 150 (15):154122, apr 2019. ISSN 0021-9606. doi: 10.1063/1.5090222. URL https: //doi.org/10.1063/1.5090222.

[92] Ivan S Ufimtsev and Todd J Martínez. Quantum Chemistry on Graphical Processing Units. 1. Strategies for Two-Electron Integral Evaluation. Journal of Chemical Theory and Computation, 4(2):222-231, feb 2008. ISSN 1549-9618. doi: 10.1021/ct700268q. URL https://doi.org/10.1021/ct700268q.

[93] Ivan S Ufimtsev and Todd J Martinez. Quantum Chemistry on Graphical Processing Units. 2. Direct Self-Consistent-Field Implementation. Journal of Chemical Theory and Computation, 5(4):1004-1015, apr 2009. ISSN 1549-9618. doi: 10.1021/ct800526s. URL https://doi.org/10.1021/ct800526s.

[94] Ivan S Ufimtsev and Todd J Martinez. Quantum Chemistry on Graphical Processing Units. 3. Analytical Energy Gradients, Geometry Optimization, and First Principles Molecular Dynamics. Journal of Chemical Theory and Computation, 5(10):2619-2628, oct 2009. ISSN 1549-9618. doi: 10.1021/ct9003004. URL https://doi.org/10.1021/ct9003004.

[95] P Jeffrey Hay and Willard R Wadt. Ab initio effective core potentials for molecular calculations. Potentials for the transition metal atoms Sc to Hg. The Journal of Chemical Physics, 82(1):270-283, jan 1985. ISSN 0021-9606. doi: 10.1063/1.448799. URL https: //doi.org/10.1063/1.448799.

[96] Jimmy Charnley Kromann. Calculate Root-mean-square deviation (RMSD) of Two Molecules Using Rotation, GitHub, v1.3.2, 2020. URL https://github.com/charnley/ $\mathrm{rmsd} / \mathrm{releases} / \mathrm{tag} / \mathrm{rmsd}-1.3 .2$.

[97] W Kabsch. A solution for the best rotation to relate two sets of vectors. Acta Crystallographica Section A, 32(5):922-923, sep 1976. ISSN 0567-7394. URL https: //doi.org/10.1107/S0567739476001873.

[98] Michael W Walker, Lejun Shao, and Richard A Volz. Estimating 3-D location parameters using dual number quaternions. CVGIP: Image Understanding, 54(3):358-367, 1991. ISSN 1049-9660. doi: https://doi.org/10.1016/1049-9660(91)90036-O. URL http: //www.sciencedirect.com/science/article/pii/1049966091900360. 
[99] Arthur Dalby, James G. Nourse, W. Douglas Hounshell, Ann K.I. Gushurst, David L. Grier, Burton A. Leland, and John Laufer. Description of several chemical structure file formats used by computer programs developed at molecular design limited. Journal of Chemical Information and Computer Sciences, 32:244-255, 2 1992. ISSN 00952338. doi: 10.1021/ci00007a012. URL https://pubs.acs.org/sharingguidelines.

[100] P.G. Edwards and R.G. Jaouhari. Synthesis and characterization of complexes of nickel(ii), palladium(ii) and platinum(ii) with the new bifunctional aminodiphosphine ligand hn(ch2ch2ch2pme2)2. Polyhedron, 8(1):25-28, 1989. ISSN 0277-5387. doi: https: //doi.org/10.1016/S0277-5387(00)86374-3. URL https://www.sciencedirect.com/ science/article/pii/S0277538700863743.

[101] Denis Spasyuk, Samantha Smith, and Dmitry G. Gusev. Replacing phosphorus with sulfur for the efficient hydrogenation of esters. Angewandte Chemie International Edition, 52 (9):2538-2542, 2013. doi: https://doi.org/10.1002/anie.201209218. URL https:// onlinelibrary.wiley.com/doi/abs/10.1002/anie.201209218.

[102] Stephan Gründemann, Martin Albrecht, Jennifer A. Loch, Jack W. Faller, and Robert H. Crabtree. Tridentate carbene ccc and cnc pincer palladium(ii) complexes: Structure, fluxionality, and catalytic activity. Organometallics, 20:5485-5488, 12 2001. ISSN 02767333. doi: 10.1021/om010631h. URL https://pubs.acs.org/sharingguidelines.

[103] Georgy A. Filonenko, Elena Cosimi, Laurent Lefort, Matthew P. Conley, Christophe Copéret, Martin Lutz, Emiel J.M. Hensen, and Evgeny A. Pidko. Lutidine-derived rucnc hydrogenation pincer catalysts with versatile coordination properties. ACS Catalysis, 4:2667-2671, 8 2014. ISSN 21555435. doi: 10.1021/cs500720y. URL https: //pubs.acs.org/sharingguidelines.

[104] Karl Z. Demmans, Maxwell E. Olson, and Robert H. Morris. Asymmetric transfer hydrogenation of ketones with well-defined manganese(i) pnn and pnnp complexes. Organometallics, 37:4608-4618, 12 2018. ISSN 15206041. doi: 10.1021/acs.organomet. 8b00625. URL https://pubs.acs.org/sharingguidelines.

[105] Marcella Gagliardo, Preston A. Chase, Sander Brouwer, Gerard P.M. Van Klink, and Gerard Van Koten. Electronic effects in pcp-pincer ru(ii)-based hydrogen transfer catalysis. Organometallics, 26:2219-2227, 4 2007. ISSN 02767333. doi: 10.1021/om060874f. URL https://pubs.acs.org/doi/abs/10.1021/om060874f.

[106] Shan Tang, Niklas Von Wolff, Yael Diskin-Posner, Gregory Leitus, Yehoshoa Ben-David, and David Milstein. Pyridine-based pcp-ruthenium complexes: Unusual structures and metal-ligand cooperation. Journal of the American Chemical Society, 141:7554-7561, 4 2019. ISSN 15205126. doi: 10.1021/jacs.9b02669. URL https://pubs.acs.org/ sharingguidelines.

[107] Vivek Sinha, Jochem J. Laan, and Evgeny A. Pidko. Accurate and rapid prediction of pka of transition metal complexes: semiempirical quantum chemistry with a data-augmented approach. Phys. Chem. Chem. Phys., 23:2557-2567, 2021. doi: 10.1039/D0CP05281G. URL http://dx.doi.org/10.1039/D0CP05281G.

[108] Satoru Hiroto, Yoshihiro Miyake, and Hiroshi Shinokubo. Synthesis and Functionalization of Porphyrins through Organometallic Methodologies. Chemical Reviews, 117(4):2910- 
3043, feb 2017. ISSN 0009-2665. doi: 10.1021/acs.chemrev.6b00427. URL https: //doi.org/10.1021/acs. chemrev.6b00427.

[109] David Dolphin. The Porphyrins V7: Biochemistry, Part B. Elsevier, 2012. ISBN 0323145612.

[110] Karl Kadish, Kevin M Smith, and Roger Guilard. The Porphyrin Handbook, Volume 3, volume 3. Elsevier, 2000. ISBN 0123932033.

[111] Robert Grubbs. Handbook of Metathesis Volume 1: Catalyst Development and Mechanism, aug 2003. URL https://doi.org/10.1002/9783527619481.

[112] Xijun Wang, Guozhen Zhang, Li Yang, Edward Sharman, and Jun Jiang. Material descriptors for photocatalyst/catalyst design. WIREs Computational Molecular Science, 8 (5):e1369, sep 2018. ISSN 1759-0876. doi: https://doi.org/10.1002/wcms.1369. URL https://doi.org/10.1002/wcms. 1369.

[113] Albert Polman, Mark Knight, Erik C Garnett, Bruno Ehrler, and Wim C Sinke. Photovoltaic materials: Present efficiencies and future challenges. Science, 352(6283):aad4424, apr 2016. doi: 10.1126/science.aad4424. URL http://science.sciencemag.org/ content/352/6283/aad4424. abstract.

[114] SeJin Ahn, Sunghun Jung, Jihye Gwak, Ara Cho, Keeshik Shin, Kyunghoon Yoon, Doyoung Park, Hyeonsik Cheong, and Jae Ho Yun. Determination of band gap energy (Eg) of Cu2ZnSnSe4 thin films: On the discrepancies of reported band gap values. Applied Physics Letters, 97(2):21905, jul 2010. ISSN 0003-6951. doi: 10.1063/1.3457172. URL https: //doi.org/10.1063/1.3457172.

[115] J S Griffith and L E Orgel. Ligand-field theory. Quarterly Reviews, Chemical Society, 11 (4):381-393, 1957. ISSN 0009-2681. doi: 10.1039/QR9571100381. URL http://dx. doi.org/10.1039/QR9571100381.

[116] Ya Zhuo, Aria Mansouri Tehrani, and Jakoah Brgoch. Predicting the Band Gaps of Inorganic Solids by Machine Learning. The Journal of Physical Chemistry Letters, 9(7): 1668-1673, apr 2018. doi: 10.1021/acs.jpclett.8b00124. URL https://doi.org/10. 1021/acs.jpclett.8b00124. 